\title{
SATURN'S CLOUD STRUCTURE INFERRED FROM CASSINI ISS
}

\author{
A Thesis \\ Presented to the Faculty of the Graduate School \\ of Cornell University \\ In Partial Fulfillment of the Requirements for the Degree of \\ Master of Science
}

by

Michael Thomas Roman

May 2012 



\begin{abstract}
Using high-resolution Cassini ISS images with wavelengths ranging from the ultraviolet to the near infrared, we have retrieved Saturn's atmospheric aerosol structure and properties for a broad range of latitudes in the southern hemisphere. The observations are consistent with two distinct layers of haze. Each layer is characterized by a vertical location, an optical depth, and a mean particle size, all of which vary with latitude. The tropospheric haze is optically thickest and extends to the greatest heights ( 40 mbar) over the equator; its top surface is at significantly greater depths $(\sim 150$ mbar ) at mid-latitudes. The height of the haze correlates well with position of the tropopause as indicated by the temperature field. Beneath this haze, we find a scattered denser cloud responsible for small-scale contrasts at an average depth of $1.75 \pm 0.4$ bar, with some features deeper than 2.5 bar.
\end{abstract}




\section{BIOGRAPHICAL SKETCH}

Michael Roman transferred into Cornell University in 2004 as a junior in the field of Atmospheric Sciences, after having studied at a small community college. As an undergraduate, he became involved in research as a student research assistant, organizing and processing image data from the Galileo mission. The project combined two of his of long-lived interest-meteorology and astronomy - and encouraged him to pursue further research experience. After receiving his Bachelor of Science degree from the College of Agriculture and Life Sciences in 2006, he continued working as a research assistant under the supervision of Professor Peter Gierasch and Dr. Don Banfield. After two years of work, he enrolled as a graduate student in the field of Atmospheric Sciences at Cornell University, with his previous supervisors serving as his academic advisors. As a graduate student, he analyzed Cassini image data, investigating clouds and hazes on Titan, Jupiter, and Saturn. The results of his analysis of the vertical structure of aerosols in Saturn's atmosphere are presented here. 
To those who have tolerated my endless fussing, reworking, second-guessing, and excessive yet fallible caution - thank you for your patience and please accept this dedication as my apology. 


\section{ACKNOWLEDGMENTS}

This work would not been possible without the expert guidance and patient support of my Special Committee, particularly my advisors and coauthors, Don Banfield and Peter Gierasch. The author wishes to acknowledge the support of the NASA Cassini project and NASA's Cassini Data Analysis Program during the period in which this work was conducted. I thank Bob West for providing guidance with the Cassini ISS filter transmissions and image calibration software, and I appreciated his thoughtful support throughout the preliminary challenges. I also thank Ashwin Vasavada for his repeated assistance with image navigation, mapping, and evaluation; Paul Helfenstein for his counseling on calibration uncertainties; and Leigh Fletcher for sharing his temperature data. Finally, I owe great thanks Santiago Pérez-Hoyos and Agustín Sánchez-Lavega for helping to verify my radiative transfer calculations by means of comparative tests. 


\section{TABLE OF CONTENTS}

BIOGRAPHICAL SKETCH......................................................................................iii

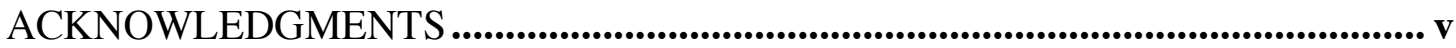

TABLE OF CONTENTS ........................................................................................... vi

LIST OF FIGURES ..................................................................................................... vii

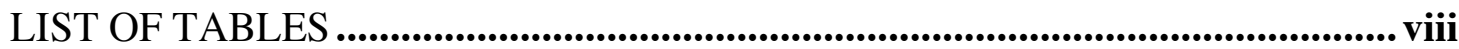

1. Introduction ................................................................................................................... 1

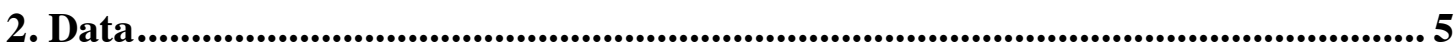

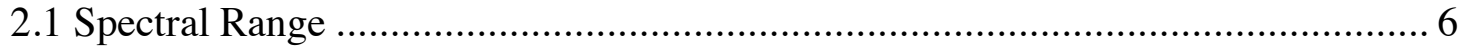

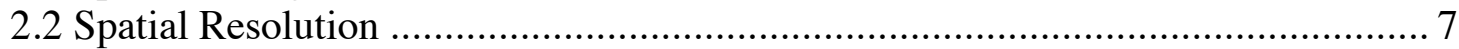

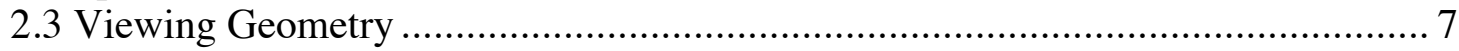

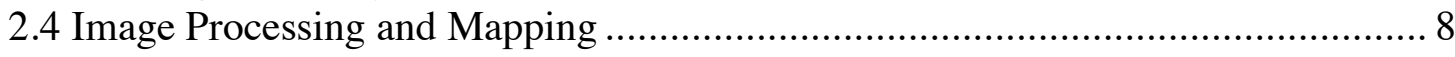

3. Method of Analysis........................................................................................................... 11

3.1 Generic Model Structure ……………............................................................ 11

3.2 Gas Properties, Scattering, and Absorption ......................................................... 13

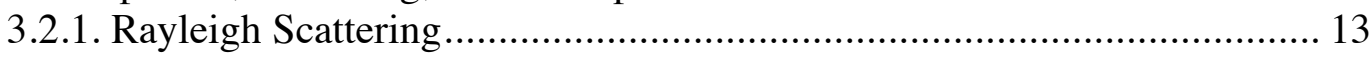

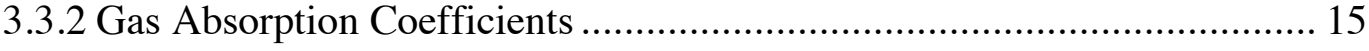

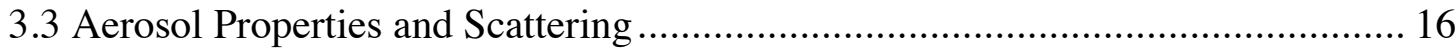

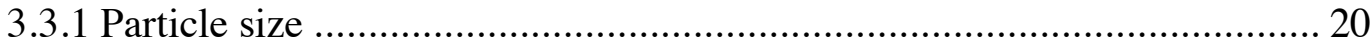

3.3.2 Single Scattering Albedos ....................................................................... 21

3.4 Model Calculations and Retrieval Technique..................................................... 21

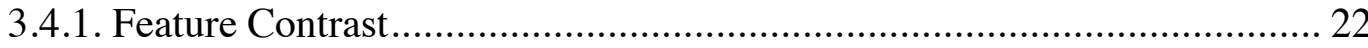

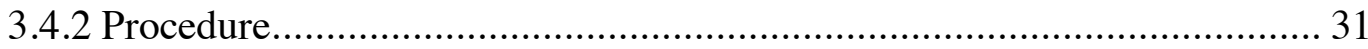

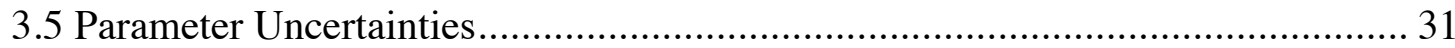

4. Results and Discussion .............................................................................................. 36

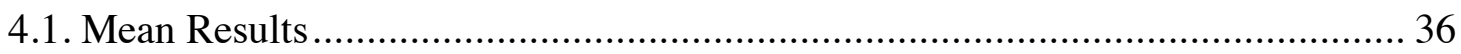

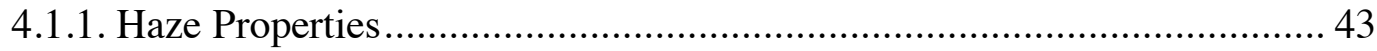

4.1.2. Cloud Properties ................................................................................. 47

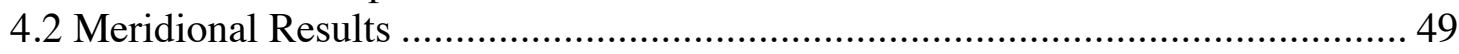

4.3 Cyclone59

5. Conclusions.............................................................................................................62

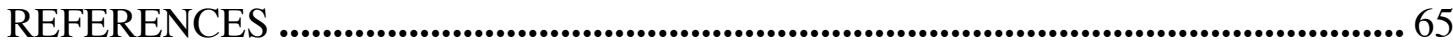




\section{LIST OF FIGURES}

Figure 1. Extinction efficiencies as a function of particle radius for each filter ......... 18

Figure 2. Asymmetry parameter as a function of particle radius for each filter.......... 19

Figure 3. Feature example along with contrast correlation and inferred structure .... 26

Figure 4. Expected contrast due to a cloud as a function of the cloud height ............ 29

Figure 5. The mean vertical structure in context with a mean temperature profile .... 43

Figure 6. Number density and corresponding visibilities for hazes............................ 46

Figure 7. Particle fall speeds as a function of height and particle size ..................... 47

Figure 8. Schematic of retrieved vertical structure for each latitude .......................... 51

Figure 9. Retrieved parameters plotted as a function of latitude ................................. 53

Figure 10. Latiduinal and vertical haze distribution superimposed upon the

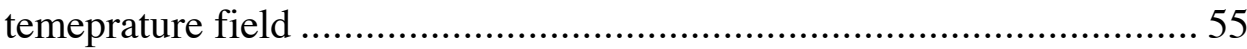

Figure 11. Haze optical depths, heights, and dT/dp as a function of latitude ............58

Figure 12. Fall times as a function of pressure and latitude for retrieved particles.... 59

Figure 13. Cyclone at $51^{\circ} \mathrm{S}$ along with schematic of retrieved structure ................... 62 


\section{LIST OF TABLES}

Table 1. Filter transmission coefficeints and wavelength single scattering albedos ... 14

Table 2. Retreived paramter mean values and standard deviations ........................... 40 


\section{Introduction}

Observations of Saturn's atmosphere show muted regional bands marked with subtle discrete features. Sunlight passing through the atmosphere is scattered and absorbed by gases and aerosols to produce the characteristically nebulous appearance-far more subdued than the dramatic features of Jupiter. The amount of scattering and absorption that light endures depends upon the abundance of aerosols as well as the wavelength of the radiation. Certain wavelengths interact with the gaseous molecules and aerosol particles more effectively than others; consequently, the depth to which light effectively penetrates and escapes the atmosphere is a function of wavelength. By exploiting this wavelength dependence, it is possible to infer qualities of the aerosols and their vertical distribution within the atmosphere. In this paper, we present the results of one such analysis; applying a combination of techniques, we investigate Saturn's vertical cloud structure over a wide range of regions at a greater spatial resolution than previously attempted.

The vertical distribution and characteristic properties of the aerosols that form the hazes and clouds are evidence of the environments in which they form. Regional, coherent variations in these environments can help reveal much about the dynamics and chemistry that shape them. The aerosols serve as tracers for winds and motions, providing clues of circulation and energy transport; identifying at which levels these tracers reside is critical to their utility. Additionally, aerosols influence the energy budget by affecting how solar radiation is distributed within the atmosphere.

Thermochemical equilibrium models of the Saturn atmosphere, as initially 
developed by Lewis (1969) and Weidenschilling and Lewis (1973), suggest vertical structures based on the lifted condensation levels of condensable atmospheric constituents. Assuming a near solar abundance of a given volatile condensable, clouds of ammonia, ammonium hydrosulfide, and water are predicted to exist at temperatures corresponding to pressures less than approximately 1.5-1.8 bar, 4.5-5.75 bar, and more than 12 bar, respectively (Atreya et al., 1999). The reality is undoubtedly more complicated than these simple models, with clouds likely existing at a range of heights and potentially varying across regions. Clearly the hazes vary with latitude as is evident when comparing the highly reflective equatorial zones with the lower albedos at mid-latitudes. Likewise, the largest storms tend to form at certain latitudes (e.g. the $35^{\circ} \mathrm{S}$ "storm alley") and not others. Knowledge of the true distribution of clouds and hazes can help place rough constraints on our assumptions of volatile abundances.

Our current understanding of the Saturn's clouds and hazes is the result of theory constrained by several decades of remote sensing data. Without the benefit of in situ observations, the vertical distribution of clouds and hazes must be inferred by analyzing the scattering and absorption of radiation from aerosols and the intervening atmosphere. A number of studies incorporating data ranging from spacecraft to earthbound observations have contributed towards a developing a rough vertical picture of these atmosphere's aerosols. Commonly, two extended, diffuse layers of aerosols, identified as the stratospheric and upper tropospheric hazes, are inferred to exist above a thick cloud layer (Karkoschka and Tomasko, 2005, Sánchez-Lavega et al., 2007; Pérez-Hoyos et al., 2005; see West et al., 2009 for a review). The origin of the haze layers that define the upper troposphere and lower stratosphere remain mostly 
speculative. Chemical modeling suggests that the stratospheric haze is the result of photochemistry (Moses, 2000). The tropospheric haze may be a greater concentration of the coagulated particles that are raining down from above, or it may be a haze of volatile condensable raised from below, or perhaps a combination of the two. Increased understanding of the aerosols that comprise these hazes can help to constrain models for their formation.

The inferred vertical extent of the hazes and the height of cloud layers vary among different studies; there is some disagreement on how high the haze reaches and whether it extends down to the cloud or not. The height and optical thickness of the deep cloud itself is sometimes assumed-placed at the theoretical predicted height and assumed infinitely thick - as the overlaying haze is investigated (Sánchez-Lavega et al., 2007, Pérez-Hoyos et al., 2005); in these studies, a few discrete features were modeled as clouds above the main cloud deck, with heights around 700 mbar and above. Other studies attempt to retrieve properties of the haze and underlying clouds, but do so with a modest spatial resolution that precludes identifying variations on anything less than broad regional scales (Karkoschka and Tomasko, 2005; Temma et al., 2005). Here we attempt to retrieve properties of the haze and potential underlying cloud at both regional and local scales by using techniques that exploit the high spatial resolution and extended spatial coverage of the Cassini Imaging Science Subsystem (ISS) data sets.

A study of thermal emission spectroscopy using Cassini VIMS spectra found a majority of opacity was located deeper than the clouds detected from imaging studies; the VIMS analysis was consistent with a concentrated cloud layer centered in the 2.52.8 bar regions with additional vertically unresolved opacity above 1.4 bar (Fletcher $e t$ al., 2011). This can be consistent with the previous imaging studies if the overlying 
opacity is dominant at visible wavelengths, obscuring the deeper cloud. In this current study, we examine the heights of cloud feature, including some of the most veiled and deep cloud features visible in the ISS and compare our results with the VIMS conclusions. A comparison between the visible and thermal data is particularly interesting given the previous apparent discrepancy in the Galileo studies of Jupiter, where the Near-Infrared Mapping Spectrometer (NIMS) and the Solid State Imager (SSI) provided different estimates of the contrasting cloud heights in the Jovian atmosphere (Irwin et al., 2001; Banfield et al., 1998)

Utilizing information on reflectivity and cloud contrasts, we employ a technique similar to that used in the Galileo SSI investigation (Banfield et al., 1998; Simon-Miller et al., 2001) to retrieve atmospheric quantities by iteratively minimizing differences between model parameters and observations. While we find that our average results are generally consistent with several previous studies and theory, we find distinct regional variations that are remarkably correlated to the observed temperature field and are thus suggestive of transport dynamics. We also find evidence of convective clouds at greater depths than previously reported in other imaging studies. We first provide an overview of the observations in following section, before discussing our analysis technique and modeling in depth in Section 3. The results of our analysis are presented and discussed in Section 4, followed by a brief concluding summary in Section 5. 


\section{Data}

The retrieval technique we employed placed demands on the spectral, spatial and temporal resolution of potential data. In addition to the wide ranging spectral coverage routinely required for vertical structure retrievals, our analysis required images of sufficient spatial resolution to resolve localized cloud features. We also sought to observe each given feature at different viewing geometries over short time intervals, effectively providing observations of a single feature at multiple emission and incidence angles through the atmosphere. Finally, to further constrain our retrievals and assess individual features in context of the average regional — or zonalstructure, we sought complimentary full-disk images featuring center-to-limb coverage of corresponding latitudes.

Cassini has been orbiting Saturn since 2004. Of the approximately 70,000 currently available images of Saturn's atmosphere captured by Cassini Imaging Science Subsystem (ISS), only a few image sets satisfied all the above requirements. High-resolution studies are often designed for measuring wind speeds and lack the spectral coverage we considered necessary for reliable vertical structure retrievals (ranging from ultra-violet to near infrared); in contrast, most photometric studies rely upon observing the change in reflectivity from the planet's center to limb and do not require great spatial resolution. Of the available images, those from the initial orbit best matched our criteria. These images only provided coverage of Saturn's southern hemisphere, as Saturn's rings obscured views of the northern hemisphere. Specifically we focused on images from October 15-16, 2004 (when the spatial resolution was greatest at $\sim 27 \mathrm{~km} / \mathrm{pixel})$. Additionally, to expand our coverage to the equator, we 
included images from July 28, 2007. For complementary full-disk coverage, we used images from May 10, 2004. The timing of these observations places them during Saturn's southern summer season (following the solstice of October, 2002). A total of 128 high-resolution images were used, along with nine full-disk images. All images were captured through the Narrow Angle Camera (NAC). Detailed information on the Cassini ISS and filters can be found in Porco et al. (2004).

\subsection{Spectral Range}

The filters chosen for use in this study were designed to pass radiation at discrete wavelengths ranging from ultraviolet to near-infrared and included both continuum and gaseous absorption bands. During the initial orbit, multi-spectral mosaics were acquired in eight filters: UV3 $(343 \mathrm{~nm})$, BL1 $(451 \mathrm{~nm})$, MT1 $(619 \mathrm{~nm})$, CB1 (619 nm, straddling the $\mathrm{CH}_{4}$ absorption band), MT2 (727 nm), CB2 (750 nm), MT3 $(889 \mathrm{~nm})$, and CB3 $(938 \mathrm{~nm})$, where numbers in parentheses are the central wavelengths of the ISS system transmission through the filter. The equatorial images from 2007 included the same filters, except UV3 and BL1. The full-disk images (used for examining the full center to limb variations in reflectivity) included all the above filters plus GRN (568 nm).

The UV3 filter constrains aerosol properties of the stratosphere, while the BL1 and GRN are ideally placed for inferring the particle color and scattering properties at a range of heights. MT1, MT2, and MT3 are each located on methane absorption 
bands of different strengths - weak, moderate, and strongly absorbing bands, respectively_providing sensitivity to different vertical heights in the atmosphere. A continuum band with very little absorption (and hence capable of penetrating deeper into the atmosphere) accompanies each of these methane absorption bands. Filter transmissions details are discussed in Section 3.2.

\subsection{Spatial Resolution}

During the initial orbit, complete coverage of Saturn's southern hemisphere was captured in $2 \times 2$ image mosaics. Images were recorded at a resolution of $1024 \times 1024$ pixels for all filters save MT3; the strong methane band images were captured at half this resolution $(512 \times 512)$, the reason being that this filter is only sensitive to high altitudes where hazes are typically uniform and lack higher spatial information.

The high-resolution orbital images from October 15-16, 2004 had an average resolution of $\sim 27 \mathrm{~km} /$ pixel. The MT3 images had a native resolution of half this value, i.e., $54 \mathrm{~km} /$ pixel. The equatorial data had an average resolution of $\sim 36 \mathrm{~km} / \mathrm{pixel}$. All the full-disk images had a resolution of $\sim 159 \mathrm{~km} /$ pixel.

\subsection{Viewing Geometry}

The orbital images were captured in near-simultaneous sets using each of the filters described above. As Saturn rotated beneath, sets were taken at regular intervals 
of $\sim 1.5$ hours to provide global coverage. In order to observe a given feature at multiple viewing angles, a feature was first targeted near nadir in one image and then identified again in an image taken $\sim 1.5$ hours later as the feature approached the limb. Assuming only insignificant changes on observable scales over the elapsed 90 minutes, locations common to two consecutive images effectively provided coincident observations of the same feature at two viewing geometries. This variation in viewing geometry is crucial to constraining atmospheric models. Our sampling is akin to an abbreviated center-to-limb variation analysis, albeit using only two points on the curve, but has the added advantages of not necessarily assuming zonal homogeneity thus allowing specific features to be directly studied. This technique follows that of Banfield et al. (1998) and Simon-Miller et al. (2001). To ease any concerns that two observing angles alone failed to adequately constrain the inferred structure, a traditional center-to-limb analysis was also performed for corresponding latitudes using May 10, 2004 full-disk images; assuming little change to the regional structure in the five months that separate the images, the complementary images were used to help validate and improve the inferred structures, particularly at high altitudes where some zonal homogeneity is expected.

The high-resolution images from 2004 and 2007 had a phase angle of $\sim 70^{\circ}$ and $\sim 31^{\circ}$, respectively. The full-disk images had a phase angle of $\sim 65^{\circ}$.

\subsection{Image Processing and Mapping}

Selected image sets were photometrically calibrated using Cassini Imaging 
Science Subsystem CALibration (CISSCAL) software (version 6.3beta) as described in Porco et al. (2004). The calibration files used were the most reliable available as of 2010, and remain the accepted values at the time of this writing. Tests using earlier versions of these calibration files resulted in differences in the absolute and relative reflectivity of several filters. For example, previous quantum efficiency corrections dating from 2004 lead to reflectivity in CB3 being 5\% greater than the currently accepted value. Retrievals using the older values would require more aerosols to produce the observed reflectivity. The evolution of calibration with time should be considered when comparing results from studies done at different times.

After calibration, image sets were mapped into registered, simple cylindrical projections using the $\mathrm{MaRC}^{1}$ mapping software. Observing geometries were used to produce ancillary maps of corresponding cosines of emission, incidence and phase angles. Constant map resolutions of $0.02 \%$ pixel and $0.1 \%$ pixel were chosen to supersample the high-resolution and full-disk images, respectively.

\subsection{Region selection}

Within these maps, regions and features common to consecutive maps with different viewing angles were identified. Specific features were used to identify precisely the same location in each map, from which $0.4^{\circ}$ square (latitude by longitude) multi-spectral samples were taken. Each of these samples provided the observed reflectivity in each passband over a small region. We targeted distinct

\footnotetext{
${ }^{1}$ MaRC (Map Reprojections and Conversions) is a free $\mathrm{C}++$ library and program developed by Ossama Othman to produce map projections. Further information and software can be found at http://sourceforge.net/projects/marc/)
} 
features, primarily discrete clouds visible in continuum filters, along with the relatively bland regions immediately adjacent to these features, presumed to be representative of the ambient haze. Distinct latitudinal regions (i.e. belts and zones) as identified by albedo variation in the methane filters were also targeted, and attempts were made to find discrete features within each of these regions; for each of few regions lacking discrete features, a representative location was sampled and the precise location in the later image was estimated using the mapped coordinates, corrected for advection due to the mean wind (Vasavada et al., 2006). For each map, this was done for all available latitudes at which locations could be traced; in practice, the amount of overlap between mosaics limited observations to planetographic latitudes between $12^{\circ} \mathrm{S}$ and $64^{\circ} \mathrm{S}$ for the 2004 data. Data from 2007 allowed us to additionally investigate latitudes between $1^{\circ} \mathrm{S}$ and $11^{\circ} \mathrm{S}$ planetographic latitude. To roughly assess the zonal homogeneity, multiple samples ( $\sim 3$ on average) were taken at each latitudinal region.

Finally a single cyclone at $\sim 51^{\circ} \mathrm{S}$ was also sampled. Multi-spectral samples were taken across the feature, crossing from north to south and east to west. This vortex was notable for its size and brightness in the continuum filters.

In summary, each of these samples provided flux-calibrated observations at eight wavelengths in two different viewing geometries for each $0.4^{\circ}(\sim 380 \mathrm{~km})$ square sample. The mean reflectivities of each sample provided 16 independent I/F observations for each location. Information on the spatial distribution of reflectivity across each cut provided observations of the small-scale contrasts at each passband. Finally, to supplement these observations, center-to-limb scans of reflectivity for 
corresponding latitudes were acquired from the full disk images acquired 5 months prior.

\section{Method of Analysis}

Our analysis was based on the method employed in the Banfield et al. (1998) and Simon-Miller et al. (2001) Galileo SSI studies. The goal was to build the simplest atmospheric models whose radiative transfer solutions concurrently satisfy the observations of reflectivity and feature contrast at multiple wavelengths and viewing angles; by exploiting the wavelength and geometric dependencies in the radiative transfer equations, properties such as vertical distribution, optical depth, size, and single scattering albedo of aerosols were inferred.

In order to discriminate between viable models, some a priori assumptions on model parameters must be made, and results should be considered in the context of these assumptions. Our model assumptions and their potential implications will be clearly stated as the model is described below.

\subsection{Generic Model Structure}

To model the scattering and absorption of light, we constructed simple models representing the vertical distribution of aerosols in Saturn's atmosphere. Aerosols were distributed in plane-parallel vertical layers with uniform mixing ratios within a gaseous atmosphere. Each layer of aerosols was characterized by an optical depth (in 
this case defined at $619 \mathrm{~nm}$ ), a mean particle size (with a central gamma size distribution width of 0.1 ), bounding pressures, and retrieved aerosol single scattering albedos for the UV3 and BL1 passbands. The layers could extend between any two pressure levels, or simply form an optically thick "sheet" at a single pressure level. The parameter values and number of layers were adjustable and determined by the data. Allowable ranges for parameters were as follows: pressures were allowed to vary between 1 mbar and 10 bar; optical depths could range from zero and 50.0; mean particle radii could range between $0.01 \mu \mathrm{m}$ and $5.0 \mu \mathrm{m}$; single scattering albedo could range between zero and 0.9999 .

Beneath the aerosol layers, the model atmosphere was given an albedo to simulate the reflection from a semi-infinite, uniform and cloud-free atmosphere that is diffusely illuminated from above (Chamberlain, 1970). This albedo was a function of the gas scattering and absorption coefficients for each filter's passband.

It is important to note that in cases of multiply scattered light, as must occur in the optically thick troposphere of Saturn, direct and completely objective retrievals of unique solutions are not possible. In these cases, the scattering of light is not linearly related to the aerosol density, and different models may produce the same reflectivity (West et al., 2004). As such, we attempt to reduce the ambiguity by demanding the models to also be consistent with the observed feature contrasts, and we present the simplest models that satisfy these observations. 


\subsection{Gas Properties, Scattering, and Absorption}

An atmosphere with a helium mole fraction of $0.119 \mathrm{He}$ (Conrath and Gautier, 2000) and a methane mole fraction of $4.7 \times 10^{-3}$ (Fletcher et al. 2009) were assumed.

Due to Saturn's oblate shape, the gravitational acceleration at cloud level changes significantly with latitude; this in turn makes the scale height and the resulting attenuation a function of latitude. For example, the gravity at $1^{\circ}$ planetographic latitude is roughly $75 \%$ the value at $64^{\circ}$ latitude; since the absorption and scattering coefficients are inversely proportional to the gravity, this leads to a roughly $33 \%$ greater transmission at $64^{\circ}$ latitude. We accounted for this in our modeling computations by computing the local gravity (at 1 bar) and corresponding gas scattering and absorption coefficients for each latitude. Gravitational parameters were taken from Jacobson (2004). A temperature profile from Lindal et al. (1985) was used for all latitudes.

\subsubsection{Rayleigh Scattering}

Based on the assumed atmospheric composition, Rayleigh scattering optical depths were computed for each filter passband. The Rayleigh optical depth per bar for each filter at $30^{\circ}$ latitude are included in Table 1. As expected the UV3 (343 nm) passband is most limited by Rayleigh scattering, reaching an optical depth of $\sim 1$ upon sensing a depth of only about 300 mbar (at normal incidence and emission through an aerosol free atmosphere). In contrast, neglecting gas absorption, the CB3 (938 nm) passband can sense closer to 17 bar before being limited by Rayleigh scattering. 
Table 1. Filter Passband Transmission Coefficients ${ }^{\mathrm{a}}$, Single Scattering Albedos

\begin{tabular}{lllll}
\hline Filter name & $\begin{array}{l}\text { Central Wavelength } \\
(\mathrm{nm})\end{array}$ & $\begin{array}{c}\text { Gas Absorption, } \kappa \\
(\mathrm{bar})^{-1}\end{array}$ & $\begin{array}{l}\text { Rayleigh, } \kappa \\
(\mathrm{bar})^{-1}\end{array}$ & $\bar{\varpi}_{0}{ }^{\mathrm{e}}$ \\
& & & & \\
UV3 & 343 & 0.0000 & 1.6709 & variable \\
BL1 & 415 & 0.0009 & 0.5301 & variable \\
GRN & 568 & 0.0169 & 0.201 & $0.980 \pm 0.005$ \\
MT1 & 619 & 0.2705 & 0.1479 & $0.995 \pm 0.005$ \\
CB1 & $619^{\mathrm{b}}$ & 0.0118 & 0.1477 & $0.995 \pm 0.005$ \\
MT2 & 727 & $2.0329(63 \%), 1.0216(37 \%)^{\mathrm{c}}$ & 0.0775 & $0.995 \pm 0.01$ \\
CB2 & 750 & 0.0173 & 0.0684 & $0.998 \pm 0.001$ \\
MT3 & 889 & $12.855(60 \%), 3.6780(40 \%)^{\mathrm{d}}$ & 0.0347 & $0.998 \pm 0.002$ \\
CB3 & 938 & 0.0132 & 0.0280 & $0.995 \pm 0.005$ \\
\hline
\end{tabular}

${ }^{\text {a }}$ Values are for $30^{\circ} \mathrm{S}$ planetocentric latitude.

${ }^{b}$ The CB1 central wavelength is the mean value of the two lobes, located on each side of the methane absorption band

${ }^{\mathrm{c}}$ Two absorption coefficients were used; weighted $63 \%$ the first term, $37 \%$ the second term

${ }^{\mathrm{d}}$ Two absorption coefficients were used; weighted $60 \%$ the first term, $40 \%$ the second term

${ }^{\text {e } U n c e r t a i n t i e s ~ u s e d ~ f o l l o w ~ t h o s e ~ r e p o r t e d ~ i n ~ S a ́ n c h e z-L a v e g a ~ e t ~ a l ., ~(2007) ~}$ 


\subsubsection{Gas Absorption Coefficients}

The relative magnitude of gas absorption at different wavelengths is critical to placing constraints on the vertical structure. The amount of light that is absorbed over a given path in part determines how deeply light can penetrate the atmosphere; filters with relatively high absorption due to methane gas are limited to sensing relatively higher levels of the atmosphere.

There are three methane filters in the present Cassini set-MT1 $(619 \mathrm{~nm})$, MT2 (727 nm), and MT3 (889 nm), each of which is centered on an increasingly strong methane band. As a result, in the absence of aerosol, at $30^{\circ}$ latitude MT1 reaches an optical depth of unity at about 1.9 bar, MT2 can sense to about 340 mbar and MT3 can sense only to about 60 mbar, with values roughly $15 \%$ deeper at high latitudes and lesser at the equator. Clouds and haze deeper than these respective heights obviously still contribute to the reflectivity in the filters, but the contrast produced by discrete features will be significantly diminished at greater depths.

All other filters have passbands located outside of the methane bands and thus experience very little absorption due to methane, particularly the continuum band filters, for which there are three corresponding to the three methane filters-CB1 (centered at $619 \mathrm{~nm}$, but passing radiation on each side of the methane band), CB2 (750 nm), and CB3 (938 nm). Collision induced hydrogen absorption ads an additional but minor source of gas absorption in the CB2 band. Rayleigh optical depth essentially limits the continuum filters in the absence of aerosol. By exploiting these relative differences in the heights sensed, leverage on the vertical distribution of aerosols is gained. 
Methane absorption values were calculated using Irwin's tables of 'correlated$\mathrm{k}^{2}$ derived from methane absorption coefficients of Karkoschka and Tomasko (2010). Collision induced hydrogen absorption $\left(\mathrm{H}_{2}-\mathrm{H}_{2}\right)$ coefficients were computed from a table made available by Borysow et al., (2002).

Two-way transmission curves as a function of pressure were computed for each filter passband as a function of latitude. To facilitate transmission calculation in the radiative transfer models, exponential functions in the form of Beer's law were fit to the transmission curves. While most filters were very well modeled with this simple fit, it was found that two-term (weighted) exponentials were necessary to accurately fit the strong (MT3) and moderate (MT2) methane band transmissions. The values of absorption coefficients for $30^{\circ}$ planetographic latitude are listed in Table 1. In summary, the combination of absorption and scattering results in a transmission for each filter due to the gaseous component alone. For the methane bands, this results in a two-way optical depth (from the top of the atmosphere, down to a given pressure level, and back) of unity being reached at pressures ranging from less than 60 mbar for MT3 to slightly more than a bar for MT1. The continua reach similar optical depths at approximately 3 bar, 6 bar, and 12 bar for CB1, CB2, and CB3, respectively.

\subsection{Aerosol Properties and Scattering}

Aerosols were treated as spherical particles (to facilitate calculations) with a real index of refraction of 1.4 , as commonly chosen based on ammonia ice data of

${ }^{2}$ These table of correlated-k CH4 coefficients can be found at Irwin's homepage at http://www.atm.ox.ac.uk/user/irwin/kdata.html 
Martonchik et al. (1984). We ignored polarization and Raman scattering. For each filter channel, particle phase functions were described with a two-term HenyeyGreenstein phase function, following the results of Tomasko and Doose (1984). The two-term function has weighted forward and backward scattering terms, each with a corresponding asymmetry parameter, $\mathrm{g}_{1}$ and $\mathrm{g}_{2}$, respectively. We initially performed our calculations with a single forward scattering term but found the two term function more accurately fit the center-to-limb variations; the lack of the backscattering term also forced the need for unrealistically smaller, isotropically scattering particles to provide sufficient back-scattering.

Using a few test cases, we explored a range of values of $g_{1}$ and $g_{2}$. We found that the best fits were achieved with $\mathrm{g}_{1}$ ranging from 0.6 to 0.8 and $\mathrm{g}_{2}$ ranging from 0.2 to 0.3 , with a weighting fraction, $f$, of $\sim 0.7$ for the forward term and (1- $f) \sim 0.3$ for the backscattering term. Previous studies employing a two-term Henyey-Greenstein function found the $\mathrm{g}_{2}$ term to have an average value of $\sim 0.3$ for multiple wavelengths with only small variation, along with similar weightings (Tomasko and Doose, 1984; Perez-Hoyos et al., 2005). To reduce the number of free parameters, we fixed our $\mathrm{g}_{2}$ at 0.3 and $f$ at 0.7 and only retrieved the forward scattering asymmetry parameter, $\mathrm{g}_{1}$. We then related this value to the particle size by substituting the Mie value of $\langle\cos \alpha\rangle$ for $g_{1}$. Figure 1 shows the expected $g_{1}$ value as a function of particle size and filter central wavelength.

Similarly, based on Mie calculations, an effective aerosol optical depth at each wavelength was related to a fiducial optical depth (here chosen to be expressed at 619 $\mathrm{nm})$ by a ratio of extinction efficiencies. See Figure 2. 


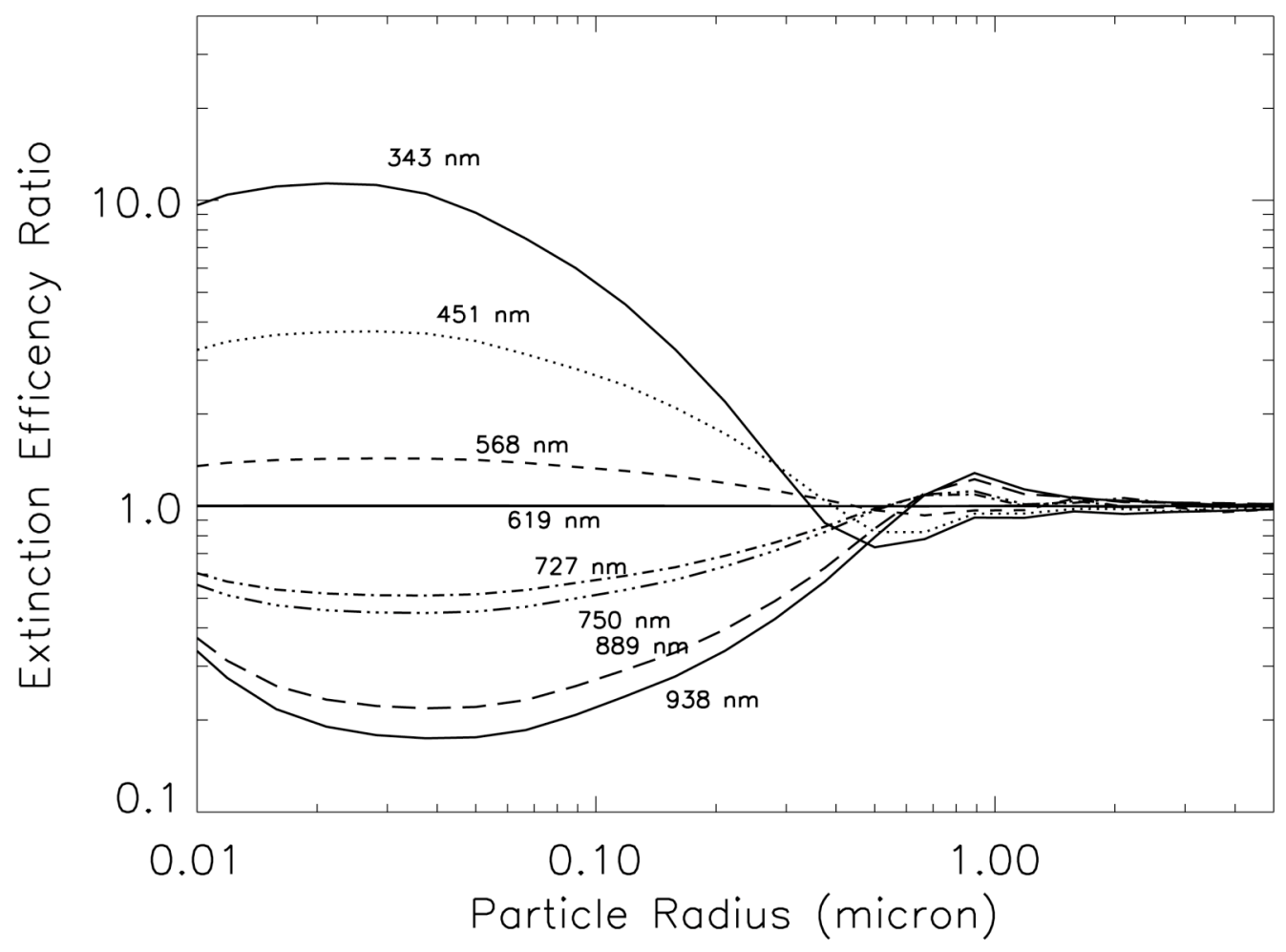

Figure 1. Extinction efficiencies as a function of particle radius for the central wavelength of each filter, expressed as a ratio to extinction efficiencies at $619 \mathrm{~nm}$. The differences in ratios are used as leverage to discriminate between different sized aerosols in the retrievals. Note that smaller radii allow for greater discrimination. 


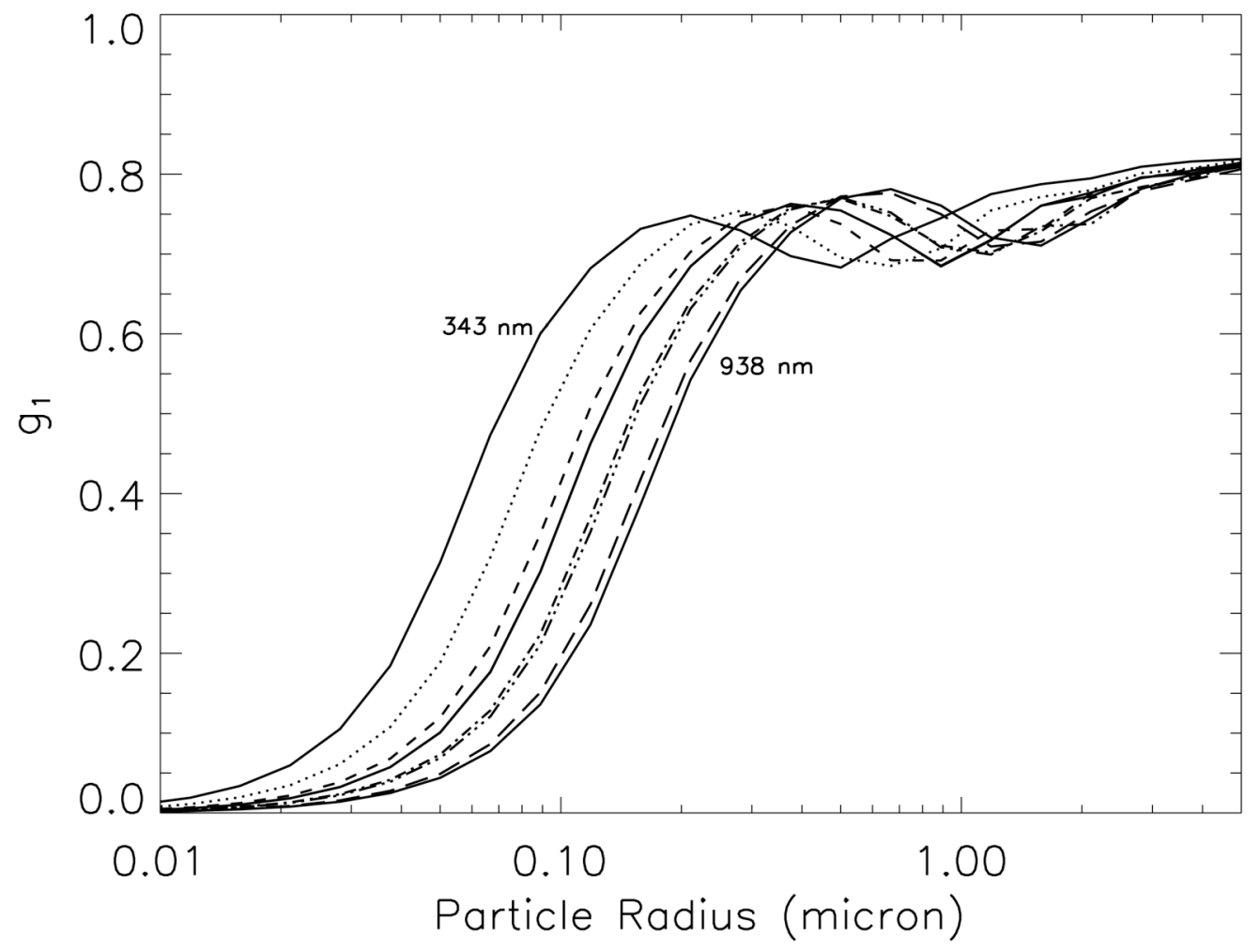

Figure 2. The asymmetry parameter that defines the forward scattering term, $g_{1}$, in the scattering phase function. Along with the extinction efficiency (Figure1), the scattering asymmetry is used to infer the aerosol sizes. 


\subsubsection{Particle size}

We attempted to constrain a mean particle size using the relationships plotted in Figure 1 and Figure 2. Figure 1 suggests that the degree of forward scattering at a given wavelength can be used to discriminate among a range of particle sizes. Likewise, Figure 2 shows that the extinction efficiency ratio is a function of both wavelength and particle size, and so the relative magnitude of aerosol optical depths at each filter, as required by the data, is also indicative of particle size. Particles of 0.02 $\mu \mathrm{m}$ radius scatter almost isotropically and exploit differences in extinction efficiencies to maximize the optical depth for the ultraviolet wavelengths while maintaining relatively lower optical depths at longer wavelengths; however, particles with a radius approaching $2 \mu \mathrm{m}$ tend to have more equal scattering properties at all wavelengths, with an asymmetry parameter around 0.75 and converging extinction efficiencies. This suggests that the smallest particles are constrained by the differences at the shortest wavelengths and largest particles are generally less constrained, i.e. only constrained to be above about $2 \mu \mathrm{m}$ in radius.

It worth noting that in the cold temperatures expected at cloud levels in Saturn's atmosphere, aerosols of ammonia would be frozen as ice crystals rather than spherical droplets. The mean sizes we retrieve are in part dependent on the phase function, described above, where the observed scattered intensity is measured at a scattering angle determined by the imaging geometry $\left(\sim 110^{\circ}\right.$ scattering angle given Saturn's $\sim 70^{\circ}$ phase angle). This is converted to a spherical droplet size, but this conversion may be sensitive to particle shape, as Karkoschka and Tomasko (2005) suggest. How the aerosol size inferred by the extinction efficiencies relates to the size 
of physical crystals with irregular shapes is not clearly understood. Interpretations of aerosols based upon these retrieved sizes should be considered in the context of such uncertainties.

\subsubsection{Single Scattering Albedos}

The impact of single scattering albedo, $\varpi_{0}$, at different levels and wavelengths was investigated. We found that our data could not independently constrain the single scattering albedo at all wavelengths while inferring optical depth. As a starting point, we chose to adopt the $\varpi_{0}$ values used by Sánchez-Lavega et al. (2007) and PérezHoyos et al. (2005) for the near-infrared filters. These values approach unity at wavelengths greater than $600 \mathrm{~nm}$ and decreased towards the ultraviolet, with greater uncertainty at shorter wavelengths. Test cases showed we could achieve good fits to the near-infrared filter data with only minor tweaks to these adopted values, over several regions and viewing geometries. To reduce our number of free parameters we fixed the near infrared wavelength single scattering albedos at these values. However, given the greater uncertainty in $\varpi_{0}$ at shorter wavelengths, and the potential for these values to vary from equator to pole (Karkoschka and Tomasko, 2005) we chose to allow the single scattering albedos at $343 \mathrm{~nm}(\mathrm{UV} 3)$ and $451 \mathrm{~nm}$ (BL1) to vary as a free parameter. We retrieved these values as the optical depths and particle sizes were concurrently constrained in part at longer wavelengths. The values used are listed in Table 1.

\subsection{Model Calculations and Retrieval Technique}

Models were iteratively constructed and evaluated using an adding-doubling radiative transfer code based on an algorithm described by Hansen and Travis (1974). 
It is an extension of the same software employed in the Banfield et al. (1998) study. Following their method, parameters were determined using an iterative least squares minimization technique. A reduced Chi-squared for each iteration was computed assuming observational errors based on NAC photometry standard deviations determined by West et al. (2010).

The method consisted of selecting multiple model parameters (generally 2 to 5) to vary within allowable ranges, as other parameters were temporarily held constant; the goodness of the fit was calculated for each combination of values, and those that best fit the data were chosen to define an improved model. This model was then further optimized as parameters in different combinations were then allowed to vary, with the best fits superseding the previous values. Through such iteration, the model was eventually optimized for a given set of observations. Beginning with a single layer, additional layers were added if required to improve the fit. Resulting values of reduced $\chi^{2}$ were typically around one or less for the best fits, indicating that our models, while still as simple as possible, were rich enough to capture essentially all of the information content in the data sets used.

\subsubsection{Feature Contrast}

Key to our technique, models are evaluated by their ability to simultaneously reproduce the observed reflectivity of each wavelength and viewing angle as well as their ability to match the correct amount of contrast observed at each wavelength within a sample. The premise is that sharp contrasts on a small scale (on order of a few hundred kilometers) are likely to due to variations in localized convective clouds. 
Such features are seen most clearly in the deeply sensing filters as opposed to the strongly attenuated UV and methane bands; the continua filters can penetrate the gas and hazes to observe these variations, whereas the same signal is dampened when observed through highly Rayleigh scattering wavelengths or through absorption bands. It follows that the cloud is relatively deep, and the relative strength of the contrast among bands that penetrate to varying depths can help to constrain the depth at which a cloud exists. By attempting to reproduce the relative contrasts caused by these discrete clouds while concurrently matching the reflectivity at multiple wavelengths and angles, we placed additional constraint upon the properties of the overlying haze and the height of the cloud.

To quantify this contrast, we examine the strength of the correlation between pairs of filters. For example, consider a spatially resolved bright feature observed through a continuum filter, with a defined increase in reflectivity, $\Delta \mathrm{I} / \mathrm{F}_{\mathrm{CB} 2}$, relative to its surroundings. When observed through a different but similarly penetrating continuum filter, this feature will also produce a similarly defined increase in reflectivity, $\Delta \mathrm{I} / \mathrm{F}_{\mathrm{CB} 1}$, relative to the surroundings. The signal produced by a true feature (as opposed to image noise) is well correlated between the two continuum filters. A plot of the observed reflectivity in one continuum versus the other would show a linear distribution. Furthermore, since the abscissa $\left(\Delta \mathrm{I} / \mathrm{F}_{\mathrm{CB} 2}\right)$ and ordinate $\left(\Delta \mathrm{I} / \mathrm{F}_{\mathrm{CB} 1}\right)$ ranges in this plot are determined by the magnitude of the contrast, the linear fit to the distribution would have a slope near unity. If the same feature is now observed through a methane filter, a correlated brightness may be observed, but the corresponding increase in reflectivity, $\Delta \mathrm{I} / \mathrm{F}_{\mathrm{MT} 1}$, would be less due to the attenuated 
signal. A plot of this change in reflectivity versus a continuum filter will still produce a linear distribution since the variation is still correlated, but the weakened signal would reduce the ordinate range and thus reduce the slope of the linear fit. Given an even more strongly attenuated filter, such as MT2, the feature would no longer be visible at all and consequently there would no correlation or well-defined slope. Based on this reasoning, we quantified the observed contrasts as a measurable change in reflectivity within a continuum channel as well as the slope of the linear fit to the correlation between this continuum and other filters.

Figure 3 shows an example of these correlation plots for a particularly distinct feature located at $30^{\circ} \mathrm{S}$ latitude and pictured here. The images are stretched to emphasize the contrast and labeled by the filter name, and presented in order of increasing sensitivity Each covers a $4.4^{\circ}$ square of latitude by longitude. The bright feature marked ' $a$ ' in the CB2 band image is not at all visible in the MT2 band, but still prominent in the MT1 band. The correlations contrast produced by this feature against the less reflective adjacent region is presented below the images.

To model these small-scale contrast variations, we assume that the observed contrast is solely due to a significant enhancement of aerosol optical depth at a single height in the atmosphere. If the cloud in reality has significant vertical extent, then this height corresponds to the height at cloud top. We assume the overlying haze is relatively uniform over the feature. An initial cloud height and optical depth were presumed and the models were then evaluated for the structure with and without the cloud; the relative differences in emergent light intensity were compared to the observed correlation between local variations in the different filter images. 


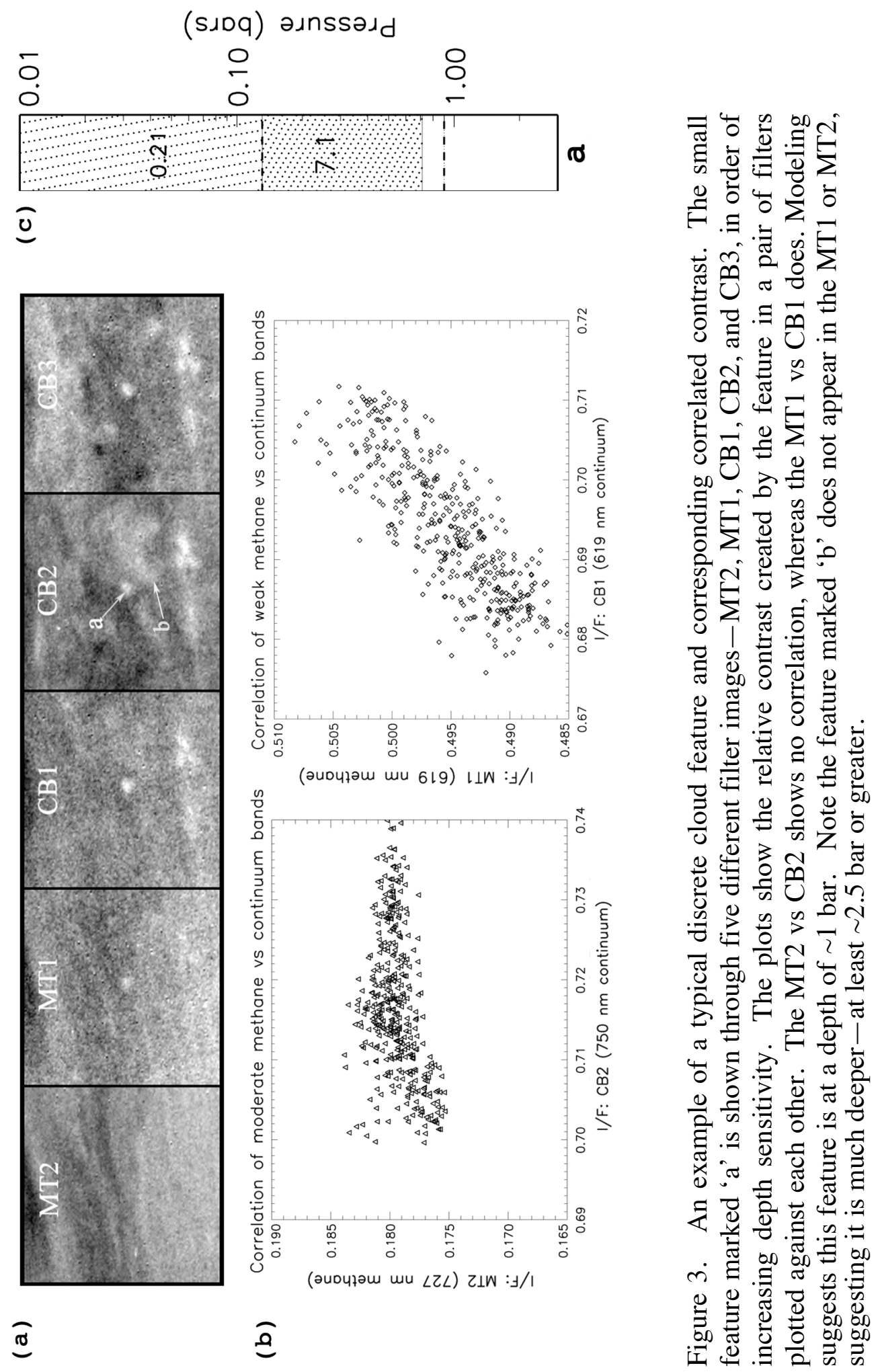


The parameters were adjusted and an optimal solution was iteratively derived along with other parameters as previously described.

For the feature marked by arrow ' $a$ ' in Figure 3, we found this contrast to occur at around 900 mbar. A schematic of the model is shown (Figure 3c) with the cloud marked as a dotted line beneath hazes, whose vertical extents and optical depths (expressed at $619 \mathrm{~nm}$ ) noted. Note that the feature marked by arrow ' $b$ ' in Figure 3 is not at all visible in the MT1 band and hardly discernable in the CB1 band, which suggests it is considerably deeper. We find that it is probably located deeper than $\sim 2.5$ bar, as will be discussed further along with other results in Section 4 .

In practice, we focused on the correlated contrast between the CB1 and MT1 channels. These filters observe at similar wavelengths and thus reduce complications due to differences in assumed single scattering albedo and scattering properties. We expect aerosol scattering to be essentially equivalent, so any differences in the contrast must be due to differences in amount of attenuation resulting from methane absorption. This is important for reducing ambiguity in determining cloud heights. A small change in the optical depth above a haze layer or a large change in optical depth deep beneath a moderately attenuating (but not opaque) haze layer can produce similar contrast in a continuum channel, but the relatively stronger attenuation in the methane band (due to the well mixed methane) leads to a much greater decrease in contrast with depth; so while a high cloud would produce comparable contrasts in both filters, the deep cloud would show much less contrast in the MT1 channel when compared to the $\mathrm{CB} 1$ channel, and this relative contrast allows us to discriminate between the otherwise ambiguous combination of $\Delta \tau$ and height. Furthermore, the differences in 
transmission between the two filters are conveniently placed to discriminate cloud heights in the roughly 1 to 2 bar region where the putative ammonia cloud is expected.

Another way of displaying this contrast information is by plotting the expected contrast a cloud would create if placed at a given height in an atmosphere (following the technique of Sánchez-Lavega et al. 2007). For example, in Figure 4, the expected contrast is plotted on the x-axis and pressure is plotted on the y-axis. An atmospheric structure was derived for a feature at $30^{\circ} \mathrm{S}$ latitude, similar to the one in Figure 3. The dotted region above 700 mbar represents the extent of $\sim 7 \tau$ of tropospheric haze in our best fitting model. The curved lines represent the expected contrasts for each filter as a function of pressure due to the presence of an optically thick cloud composed of 2 $\mu \mathrm{m}$ particles with a $\varpi_{0}$ of 0.994 . The vertical dotted line marks $1 \%$ contrast and is deemed the limit of detection. As can be seen, for this model, the moderate methane band MT2 (dashed-dot line) reaches $1 \%$ at about 600 mbar; therefore, a cloud located beneath 600 mbar would not produce a signal above the noise level in the MT2 filter. The MT1 reaches this $1 \%$ level much deeper in the atmosphere, at $1.4 \mathrm{bar}$, and the continua at yet deeper levels. The contrasts measured from the actual images for MT1 and $\mathrm{CB} 1$ are plotted as circles upon the expected curves, with error bars in the resulting pressure heights due to a $1 \%$ uncertainty in the contrasts values. The overlap between the error bars constrains the height of the feature. Plotted this way, it is clear that the shape of the MT1 contrast curve with pressure relative to the CB1 curves explains its great utility in defining the cloud height. Using our technique, best fits for this structure placed the cloud at $850 \pm 100$ mbar. 


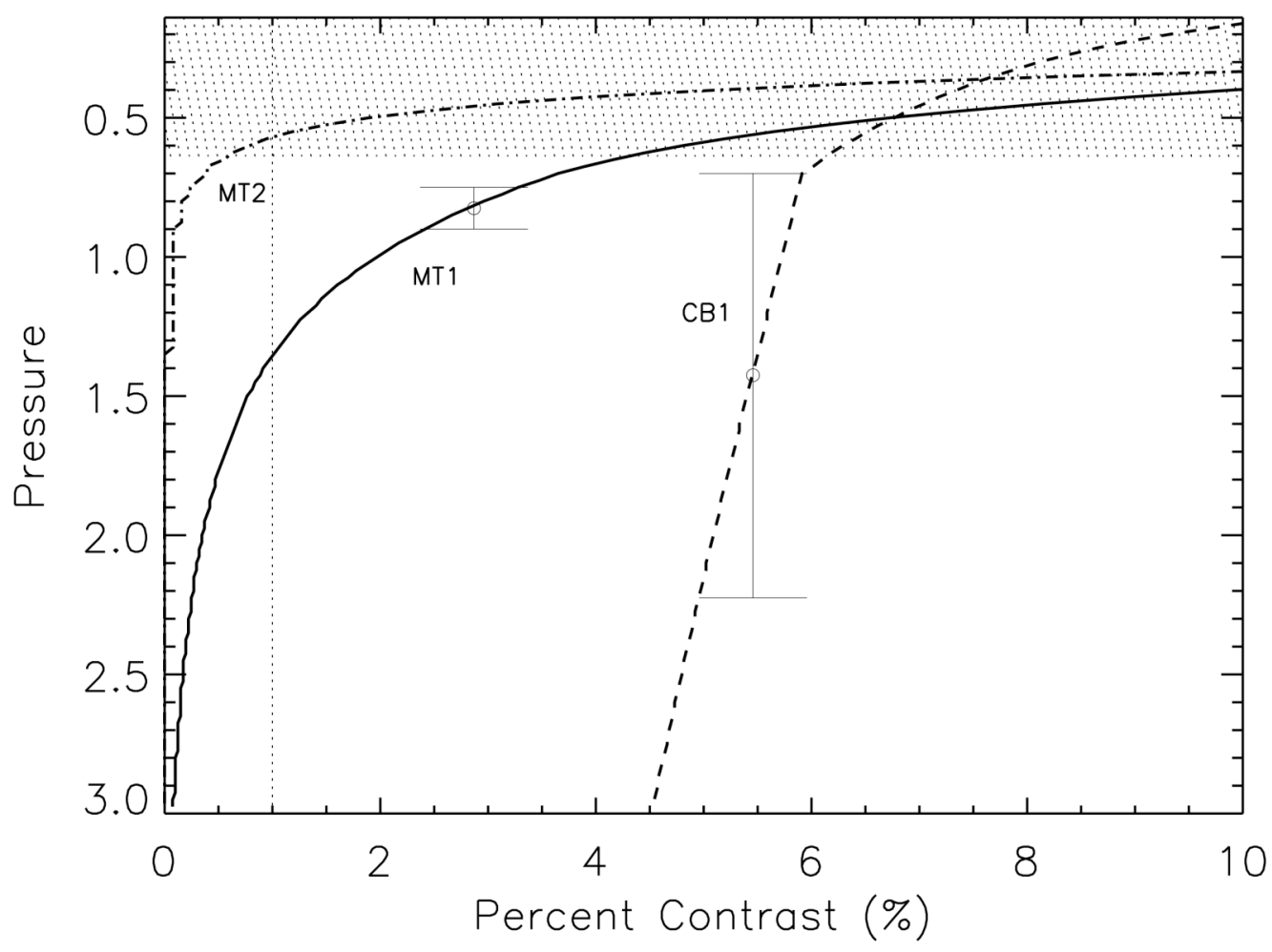

Figure 4. The expected contrasts produced by placing a discrete, optically thick cloud at different heights beneath a retrieved haze structure as plotted for several filters. The dotted region above 700 mbar represents the haze. The vertical dotted line marks the limit of contrast detection. The circles represent the observed contrast in the images and the vertical error bars show the uncertainty in contrast and consequent uncertainty in height. The strong attenuation of the MT1 provides the greatest constraint on cloud height. 
The optical thickness, mean particle size and albedo of the cloud all have impact on the expected contrast and are thus constrained as the model is developed. If the cloud is composed of perfectly white, conservative scatterers, then even a small increase in optical depth can produce a significant contrast; if the cloud is composed of non-conservative scatterers, the optical depth of the cloud can be significantly greater-even essentially infinite-before producing the same amount of contrast. The amount of reflectivity this cloud contributes is obviously also greater if the albedo of the cloud is greater, and this resulting reflectivity can be well constrained by the observations. We attempted to reduce this ambiguity by considering that some bright discrete clouds we are examining are similar to those seen in Cassini VIMS data as dark and hence optically thick to the thermal radiation $(5 \mu \mathrm{m}$ wavelength) radiating from beneath (Baines et al., 2005; Choi et al., 2009). Cloud particles of a micron or two in radius (consistent with our retrievals), have a smaller extinction efficiency at $5 \mu \mathrm{m}$ and thus require a greater optical depth (as expressed at $619 \mathrm{~nm}$ ) in order to be thick enough to block $5 \mu \mathrm{m}$ radiation. Perfectly reflective clouds of only a few $\tau$ appear incompatible with the qualitative picture that VIMS maps presents, as well being inconsistent with notions of thick condensation clouds in Earth's atmosphere. Recent analysis of VIMS spectra also suggests that the deeper clouds have optical depths of unity or greater at $5 \mu \mathrm{m}$ (Fletcher et al., 2011). The A more systematic analysis of cloud features in Cassini VIMS at both IR and near-IR wavelengths should help to resolve this ambiguity in the combination of $\tau$ and $\varpi_{0}$ and thus better constrain the optical depth of the clouds, but for our modeling, we explored both pure white clouds of low optical depth and reduced albedo clouds of essentially infinite optical 
depth. Based on the reasoning above, we strongly prefer the optically thicker clouds of reduced albedo, but present results with the caveat of this ambiguity.

The perceived contrast of a cloud is also dependent on the brightness of the background it is observed against. In our models, the gas absorption and Rayleigh scattering determine the deep atmospheric albedo. Should the deep atmosphere be darker due to an unaccounted absorber or brighter due to a layer of deeper clouds, the observed contrast would be different. It is the ratio of contrasts that is significant to our depth determination, so an absorbing or reflecting layer that affects only one of any pair of filters would cause the greatest change of the inferred height. We attempted to model some hypothetical cases to assess the model sensitivity. For example, we added $20 \tau$ of putative thick $\mathrm{NH}_{4} \mathrm{SH}$ cloud at 5 bar, which served to only slightly reduce the contrast $\mathrm{CB} 1$, while having a nearly negligible impact on the MT1, thus slightly increasing the ratio of the modeled contrasts. Clearly a large number of more complicated configurations could be constructed to alter the contrasts, but these are beyond our ability to constrain based on reflected light alone. When comparing the contrast in MT1 to CB1, it is fortunate that both of these filters become quickly attenuated below about 3 bar, so that whatever lies much deeper should have little impact on observations in these filters. Since the putative $\mathrm{NH}_{4} \mathrm{SH}$ cloud is expected to form at about 5 bar or deeper, the possible presence of this cloud has little impact on the cloud height retrieved using the combination of MT1 and CB1 filters. As such, we attempt to model all contrasts as a single layer of enhanced aerosol against an essentially cloudless atmosphere, the albedo of which is based solely upon gas absorption and scattering properties. 


\subsection{Procedure}

Our typical procedure was as follows. After identifying a feature of interest, we took a sample of the feature along with samples of immediately surrounding, indistinct region. Since our modeling assumes that small features are simply due to the presence of discrete clouds beneath or within uniform hazes, we treated the samples of the indistinct regions to be representative of the ambient atmosphere in which a cloud is imbedded. As we derived structures for each sample based on the iterative fitting technique described above, we constrained our haze layers to be consistent over the region straddling the feature $(<1000 \mathrm{~km})$. For regions where the MT2, MT3, and UV3 filters indicated the haze clearly varied on smaller scales, we relaxed this assumption and required a uniform haze over the area of the sample $(\sim 380 \mathrm{~km})$. Once a best fit was determined, we then tested how well it could reproduce the full center-to-limb variation in reflectivity for corresponding latitudes in full disk images. If center-tolimb variation could be improved by tweaking the model, we attempted to implement these adjustments and refitted the higher resolution reflectivity and contrasts until a consistent model was achieved. By these means we developed vertical structure models representative of different latitudes and features.

\subsection{Parameter Uncertainties}

Absolute, systematic uncertainty in the heights retrieved is largely due to uncertainty in methane abundance. The value used has an $\sim 5 \%$ uncertainty, leading to a similar systematic uncertainty in our retrieved heights and optical depths. Likewise, 
systematic calibration uncertainties due to uncertainties in stellar fluxes are $\sim 10 \%$, leading to similarly sized absolute uncertainties.

We found that the retrieved particle sizes were quite sensitive to the assumed phase function used. A single term Henyey-Greenstein or Mie phase function often resulted in retrieved particles sizes as much as $1 / 10$ the size of those found using the two-term function. Should future data suggest the use of a different phase function, the impact on the retrieved particle sizes should be considered.

The particle single scattering albedo is also a source of uncertainty for haze optical depths and retrieved cloud heights. Should the $\varpi_{0}$ be slightly less the hazes would be slightly thicker and more attenuating. If $\varpi_{0}$ of the haze aerosols is as low as 0.990 at $619 \mathrm{~nm}$ and $750 \mathrm{~nm}$, and slightly lower at $938 \mathrm{~nm}$, the total optical depth and base pressure of the haze can be potentially $\sim 30 \%$ greater. This would reduce feature contrasts and ultimately push clouds higher. Tests show this could result in an uncertainty of \pm 200 mbar for the height of the cloud given an uncertainty of \pm 0.005 in $\varpi_{0}$.

Larger uncertainties are due to random errors. Image noise, lapses in time between images leading to imperfect registering of images, inherent limitations due to sporadic wavelength coverage, and merely the oversimplification of the complex atmosphere that our model represents all contribute to this uncertainty. As such, the relative uncertainty of other reported parameters were determined based on the repeatability of fits and the sensitivity expressed by perturbing each parameter. As would be expected, the sensitivity to parameter changes was less for thinner hazes but amplified in the multiply scattering thicker hazes. Likewise, sensitivity to the deepest 
layers is reduced by the obscuring presence of the overlying haze.

Of the two haze layers consistently required, relative optical depths were estimated to be accurate to within $\pm 1 \tau$ for the thick, extended haze, which we refer to as the upper tropospheric haze, and as much $\pm 0.05 \tau$ for the thinner upper layer, referred to as the stratospheric haze. Particle single scattering albedos are within \pm 0.02 for the thicker, tropospheric haze as reported for BL1, but up to \pm 0.04 for the stratospheric haze at the same wavelength.

The single scattering albedo at UV3 is less constrained. In the stratosphere, it is strongly coupled with the thin stratospheric optical depth, which is only truly significant and constrained at the shortest wavelengths (as evident by the relative extinction efficiencies for the small particles in the stratospheric haze). Within the tropospheric haze, the stratospheric haze above significantly diminishes our data's sensitivity to the UV3 single scattering albedo. From our tests, we determine an uncertainty of \pm 0.1 in the UV3 single scattering albedo for both layers.

The vertical extent of the hazes and the sharpness of the boundaries were also investigated. The uppermost extent of the hazes is constrained by the amount of limb brightening in the UV3 filter. Rayleigh scattering in clear gas quickly increases reflectivity at this passband, while aerosol absorption due to low single scattering albedos quickly reduces it. A layer of clear gas above the absorbing aerosols results in higher reflectivity towards the oblique limb views, and the center-to-limb variation in this reflectivity is in part shaped by the height of the top of the stratospheric haze. The best fits were typically found at about 10 mbar for all latitudes, but this could be moved up or down 5 mbar with little change to the fit. 
The pressures of the interface between the stratospheric and upper tropospheric hazes are well constrained with a relative uncertainty estimated to be less than \pm 20 mbar. Whereas it beyond our ability to resolve distribution on length scales of less than a scale height (and hence define precisely how sharp the boundary is), we find that relative differences of only 20 mbar have a significant impact on the modeled reflectivity in MT3 and UV3. Hence regional differences in the height of the upper extent of the tropospheric haze are well constrained and significant.

The base of the upper tropospheric haze was more poorly constrained and considered to have an uncertainty of \pm 150 mbar. This uncertainty comes with the caveat that it is based on the assumption of a constant mixing ratio. The consequence of this assumption is that the extent of the layer is partially constrained by the concentration at the top of the layer. If the top of the layer needs to be optically dense in order to produce the correct reflectivity in filters sensitive to this pressure range, then the imposed constant mixing ratio assumption forces the extended layer to maintain this optical density to greater depths. This constant $\tau$ bar $^{-1}$ is effectively extended down until the total optical depth of the layer is great enough to sufficiently brighten the deeply sensing filters. The actual distribution of aerosols with height deep within this layer may vary, for only the top of the layer is well constrained; the actual mixing ratios could potentially become less at depth, thus allowing the haze to extend much further without the total reflectivity exceeding the observed reflectivity. Discriminating between these configurations is beyond the reach of our data. The assumption of a constant mixing ratio throughout the layer potentially places an artificial limit on the deeper extent of the haze, and our reported values should be 
considered with this in mind. There is simply not enough information available to infer any change in distribution throughout the depth of the layer, and the assumption is as reasonable as any other for this type of modeling.

We tested the impact of this assumption on the retrieved heights of the convective cloud beneath. We found that a more extended, diffuse layer at the base of the haze, chosen to preserve the optical depth, tended to increase the cloud altitude by up to 200 mbar. This compounded by uncertainties in properties of the overlying haze, contrast determination, and deep atmosphere properties leads to an absolute uncertainty estimated to be less than \pm 400 mbar.

As discussed, the particle sizes of the aerosols are best constrained when they are less than about $2 \mu \mathrm{m}$ in radius. Our wavelength range provides little discrimination above that. Furthermore, the equatorial data was lacking the UV3 and BL1 filters, so the ability to discriminate particle sizes was weaker for that region. As such, we find the particle sizes uncertainties for the smaller particles of the stratosphere to be \pm 0.03 , but potentially much larger as the particles become greater than $2 \mu \mathrm{m}$ in radius. Gauging how small adjustments in the particle size affect the resulting fits, we estimate the uncertainty to be about $20 \%$ for particles under this roughly $2 \mu \mathrm{m}$ threshold. For the deep cloud, only the longest wavelengths can provide any information and so the particle size is even less constrained. Presumably, these cloud particle sizes can be quite large and thus fall beyond our ability to constrain. As such, we simply report the mean value of the marginally best fits with the caveat that these particles may be significantly larger. 


\section{Results and Discussion}

\subsection{Mean Results}

As in a majority of other studies (reviewed by West et al., 2009), two layers of hazes were needed to match the observations for all locations. The top-most aerosol layer, which we identify as the stratospheric haze, is optically thin and composed of small particles. In our simple models, we find that this layer extends from $10 \pm 5$ mbar down to where it meets the next layer at $\sim 100$ mbar. This second layer has considerably greater optical depth, vertical extent, and particle sizes. We identify this layer as the tropospheric haze. The boundary between these two layers marks an order of magnitude increase in particle size and optical depth per bar of pressure. Both layers have significant absorption towards the blue. Mean values of the model parameters, as computed from all retrievals $(\sim 100)$ ranging from the equator to $64^{\circ} \mathrm{S}$ latitude, with standard deviations are listed in Table 2.

A cloud was needed to create the observed contrast that defines the small-scale features. The location of this cloud was defined at a discrete pressure level, on average between 1 bar and 2 bar. If the cloud were assumed to have essentially infinite optical depth, its particles required an average $\varpi_{0}$ of .993 for an albedo of $\sim 0.85$; if, on the other hand, we were to assume it was a perfectly white, its optical depth was on average only about 4 . We note that this cloud was only required to match the reflectivity and contrast for defined features; it was scattered and broken as opposed to forming a uniform layer. For the most indistinct locations, thick hazes alone could provide sufficient reflection to match the observations; however, the more frequent 
patchiness seen in VIMS maps (Choi et al. 2009) as compared to the deep contrast identifiable is ISS images, may suggest a majority of the opacity seen in VIMS data are due to clouds located at depths greater than the ISS can easily sense (i.e. $>\sim 2.5$ bar). Furthermore, for a majority of regions sampled, a very thick, uniform, white cloud at pressures less than 2.5 bar would create reflectivity irreconcilably greater than we observe at continua wavelengths. This is consistent with the results of Cassini/VIMS night-side spectra analysis that suggests a possible concentrated cloud centered in the 2.5-2.8 bar region (Fletcher et al., 2011).

We note that our best fits do not call for a clear layer between stratospheric and tropospheric hazes as many other studies have found (e.g., Stam et al., 2000, PerezHoyos et al., 2005, Sánchez-Lavega et al. 2007). Such a layer would most affect the observations in UV and strong methane bands, but we did not find that it was required to match our observations. Wavelengths shorter than those used in this data set (i.e. < $343 \mathrm{~nm}$ ) are likely more sensitive to this potential clear layer and would potentially constrain its presence or absence better than can our data set.

In aggregate, our mean results are in good agreement with results found in the literature. The retrieved stratospheric and tropospheric particle sizes are in excellent agreement with the respective $0.1-0.2 \mu \mathrm{m}$ and $1-2 \mu \mathrm{m}$ radii reported by several recent studies (Karkoschka and Tomasko, 2005; Pérez-Hoyos et al., 2005; Muñoz et al., 2004, Temma et al., 2005). The upper extent of the stratospheric haze shows less consensus among studies, but our $\sim 10$ mbar value is consistent with Sánchez-Lavega et al. (2007), Temma et al. (2005) and the chemical models of Moses (2000). The upper extent of the thicker, tropospheric haze is consistent with the roughly agreed 
value ( 100 mbar) in all of the above studies. The single scattering albedo at UV3 found here is consistent with the $0.6 \pm .1$ values reported by Karkoschka and Tomasko (2005), and Pérez-Hoyos et al. (2005).

The retrieved optical depths for our models are roughly consistent with the values found in the literature. The stratospheric haze mean value of $\tau \sim 0.08$ expressed at $619 \mathrm{~nm}$ is in excellent agreement with the value reported by Sánchez-Lavega et al. (2007) for their analysis of Cassini images; however, our tropospheric hazes optical depths fall towards the lower values they reported but agree reasonably well with the $\tau$ 10 reported for mid-latitudes by several others (Karkoschka and Tomasko, 2005; Pérez-Hoyos et al., 2005). Hazes of any greater optical thickness would have resulted in excessively high reflectivity as compared to our observations. The differences between Cassini studies (Sánchez-Lavega et al., 2007) can largely be due to changes in image calibration files in the intervening years between the studies. Greater inconsistencies between other studies could be due to seasonal changes the haze optical depths and haze scattering assumptions. Our assumed single scattering albedos (based on consistent results between Karkoschka and Tomasko, 2005; Pérez-Hoyos et al., 2005 and Sánchez-Lavega et al., 2007 and initially tweaked to optimize our fits) and calculated deep atmospheric albedos would have to be significantly reduced at nearly all wavelengths-particularly the deeply sensing, near-infrared continua wavelengths - to accommodate significantly thicker hazes and would require a worse fit to our data. Single scattering albedos less than the ranges given in Table 1 could permit higher optical depths $(>15 \tau)$, but note that the relative latitudinal changes described below would be preserved. 


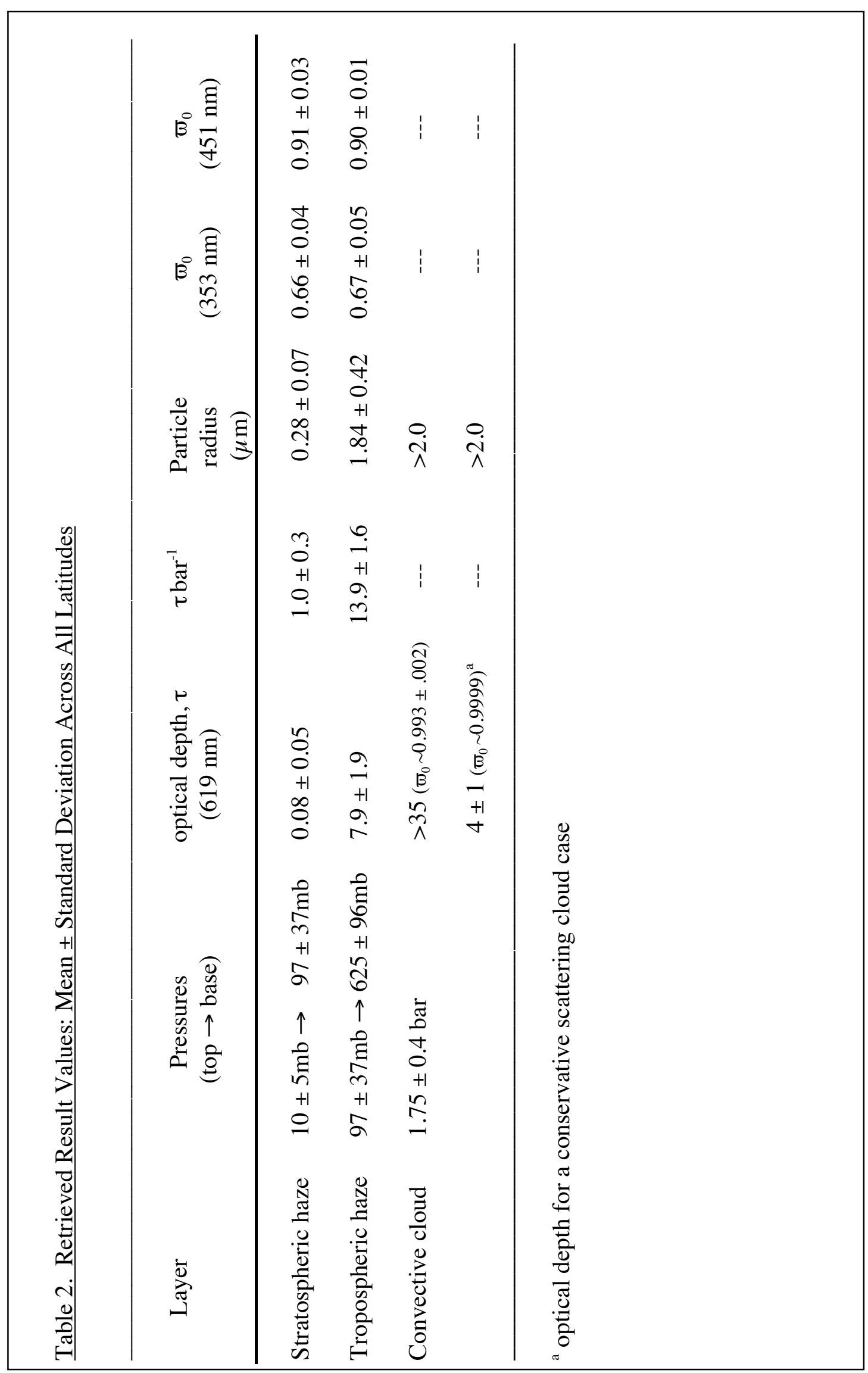


The base of the tropospheric haze in our models does not extend down to the condensate cloud as it does in some studies (Karkoschka and Tomasko, 2005; Sánchez-Lavega et al., 2007), but rather is truncated around half a bar, similar to what was found by Pérez-Hoyos et al. (2005) and Muñoz et al. (2004). However, it is worth noting that the base of this layer was not strongly constrained by the data intrinsically. As discussed above, it is required in order to maintain the constant mixing ratio $\left(\tau\right.$ bar $\left.^{-1}\right)$ - as needed at the top of the layer - throughout the entire layer while not exceeding the required total optical depth of the layer. If such a layer extended to the cloud (the height determined by the relative contrasts between MT1 and CB1), it would typically over-brighten the modeled reflectivity. If the constant mixing ratio assumption is relaxed and the tropospheric haze is broken into two separate layers such that the total optical depth is preserved, the $\tau$ bar ${ }^{-1}$ may be reduced at the base allowing a considerably optically thinner layer to fill this gap. In the interest of retrieving the simpler model, we prefer a single tropospheric haze, but it is as much constrained by the modeling limitations as by the data to be so. As discussed, the thinning, deeper haze would tend to raise the deep cloud in height by roughly 200 mbar in some cases.

Though this base pressure is sensitive to the model assumption regarding the uniformity of the layer, a physical argument can be made in its favor. When evaluated in context of the temperature profile (see Figure 5), the average base pressure occurs towards the bottom of the radiative-convective boundary, at roughly 600 mbar 
(Fletcher et al., 2007). Below this height, convective processes and eddy mixing could conceivably prevent or erode stable haze layers. This is also seen in the rapid order of magnitude increase in the eddy diffusion coefficient that occurs at this height (Moses, 2000). Similarly, the increasing stability towards the tropopause may be responsible for the upper boundary of the thicker haze layer as well. 


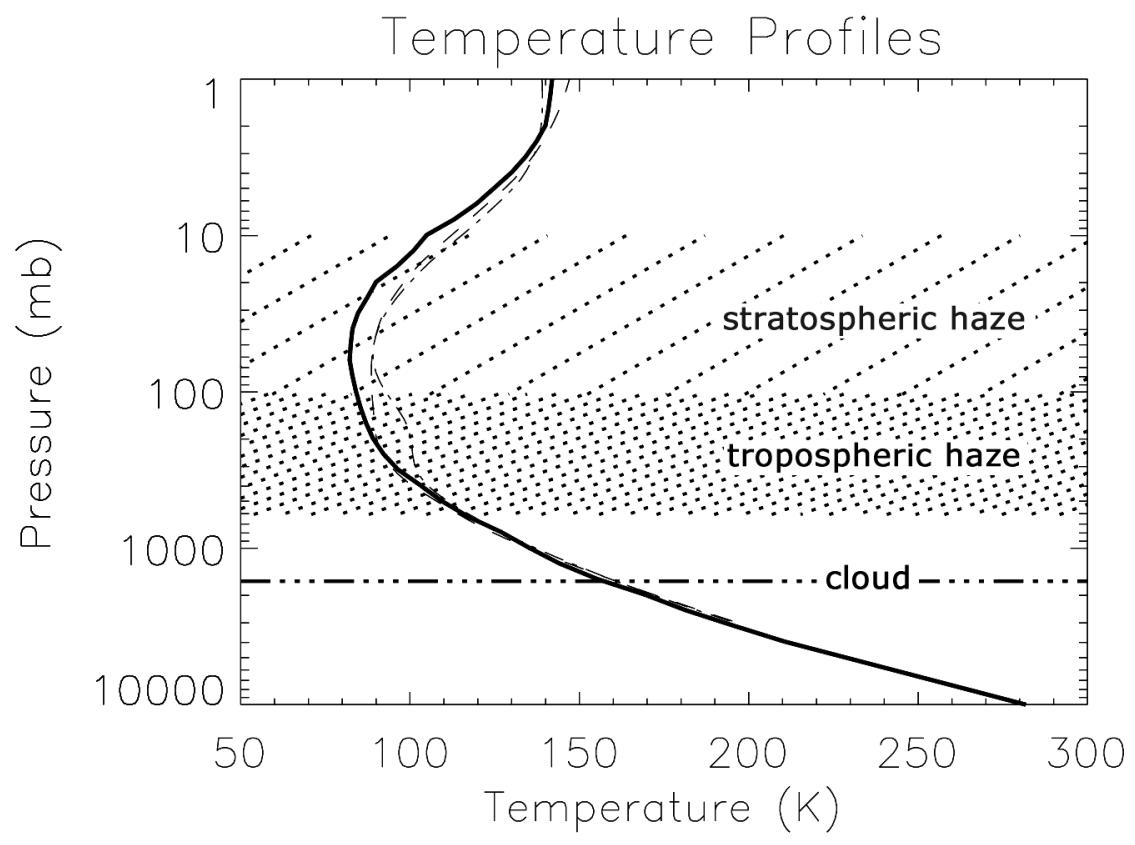

Figure 5. The mean vertical structure superimposed upon the temperature profile. The solid bold line is the temperature profile from Lindal et al. (1985); the dotted profiles are from Fletcher et al. (2009) for two different latitudes (equatorial and mid-latitude). 


\subsubsection{Haze Properties}

From our retrieved tropospheric optical depths and particle sizes, we calculated aerosol mixing ratios with a mean value of $1.5 \times 10^{-19}$ and $4.3 \times 10^{-19}$ for the tropospheric and stratospheric hazes, respectively. This yields a rough mean number density of $<6 \mathrm{~cm}^{-3}$ throughout the hazes, varying with latitude. To put this into perspective, neglecting Rayleigh scattering, the number densities would translate into analogous horizontal visibilities of over $1000 \mathrm{~km}$ in the stratospheric haze, quickly dropping to $\sim 60-100 \mathrm{~km}$ at the top of the tropospheric haze, decreasing to $\sim 20 \mathrm{~km}$ at the base; even the tropospheric visibilities would be considered excellent to very good in analogous observations for most locations on the Earth's surface, hence our use of the term haze for both of these upper aerosol layers on Saturn. Plots of number density and visibilities for two extreme cases are plotted in Figure 6. With the transition from the stratospheric to tropospheric hazes, the order of magnitude decrease in visibility that occurs over the range of a scale height or less helps illustrate how the regional contrasts we observe in the weakly penetrating methane filters can occur.

What maintains the hazes is still a matter of speculation. Photochemistry is the most likely source of aerosols in the stratosphere, but the upper troposphere may be a combination of chemical products falling from above mixing with aerosols raised from below (Moses, 2000). The flux of material from above would presumably be 
controlled by photochemical production and how quickly they fall, determined by the coagulation, coalescence, and sedimentation rates (e.g., Stam et al., 2001).

We examined the sedimentation rates for our retrieved particles over the full range of pressures for our retrievals. Figure 7 shows the sedimentation rates for spherical particles, assuming they have a density of $\sim 0.8 \mathrm{~g} \mathrm{~cm}^{-3}$ (based on the density of ammonia ice). Particles of our mean stratospheric size $(\sim 0.28 \mu \mathrm{m})$ would fall at a rate of order $0.1 \mathrm{~mm} / \mathrm{sec}$, while our tropospheric particles $(\sim 1.8 \mathrm{um})$ would fall at rate of several millimeters per second. Neglecting coagulation and coalescence, this suggests particles would take decades to fall a single scale height in stratosphere, but on the order of only a years or less for tropospheric particles. In reality, presumably irregular crystals would have more aerodynamic drag and lower density slowing down the fall rates, but coagulation and coalescence could help increase rates. Vertical velocities due to meridional circulation are expected to be very small, on order of $10^{-5}$ $\mathrm{m} \mathrm{sec}^{-1}$ (Conrath et al., 1990; West et al., 1992), and thus are incapable of suspending particles or significantly increasing the fall times. If the aerosols that compose the tropospheric haze are partly supplied by condensing volatiles from below, then penetrating convection or eddy diffusion would seemingly have to replenish the tropospheric haze on the order of a year or less and over a range of latitudes. 


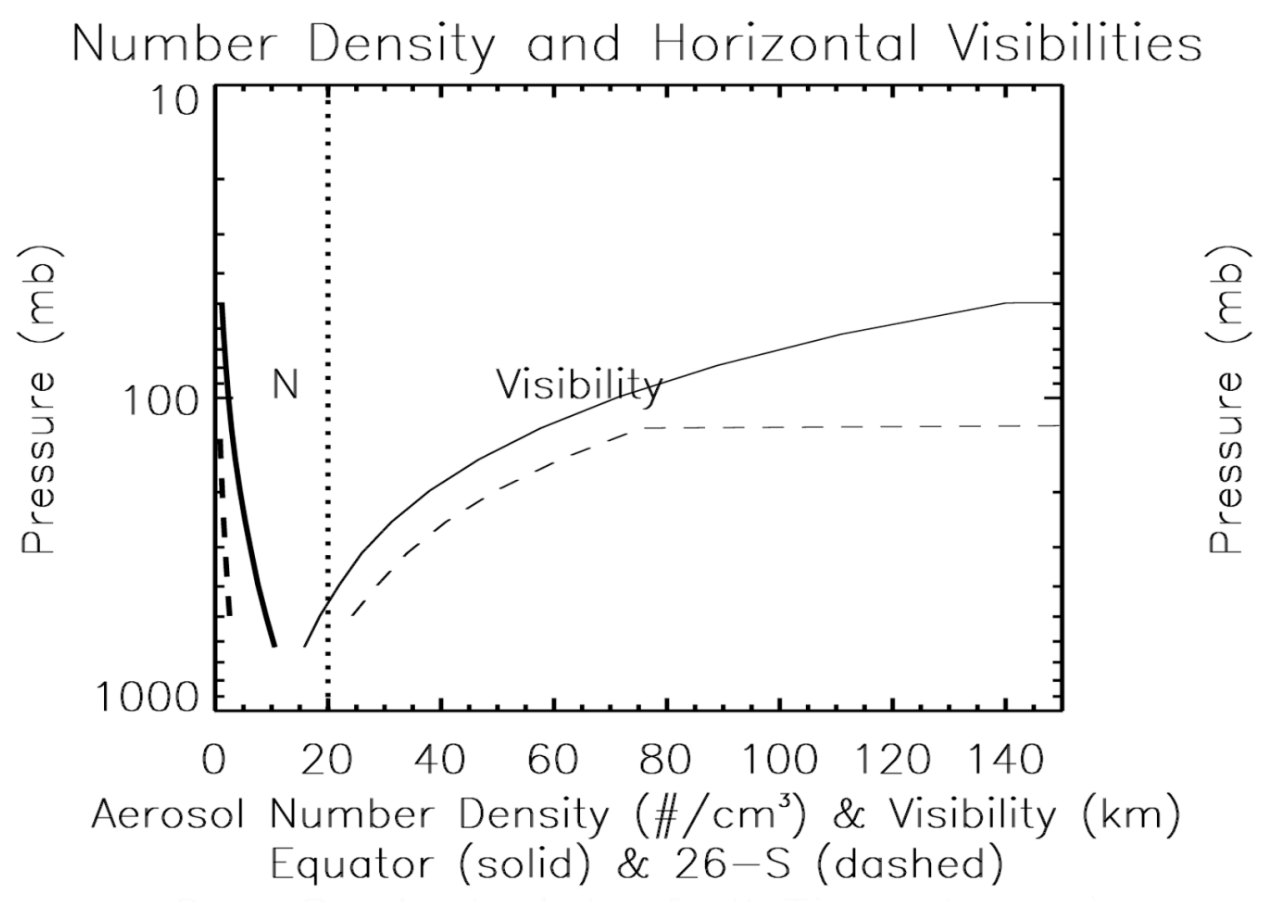

Figure 6. Number density and resulting visibility for high $\tau$ equatorial (solid) and low $\tau$ mid-latitude (dashed) regions plotted as a function of pressure. The sudden drop in visibility marks the top of the tropospheric haze layer. This decrease occurs at a greater height for the equatorial region illustrating the higher reflectivity and differing opacities as seen in the stronger methane band images. 


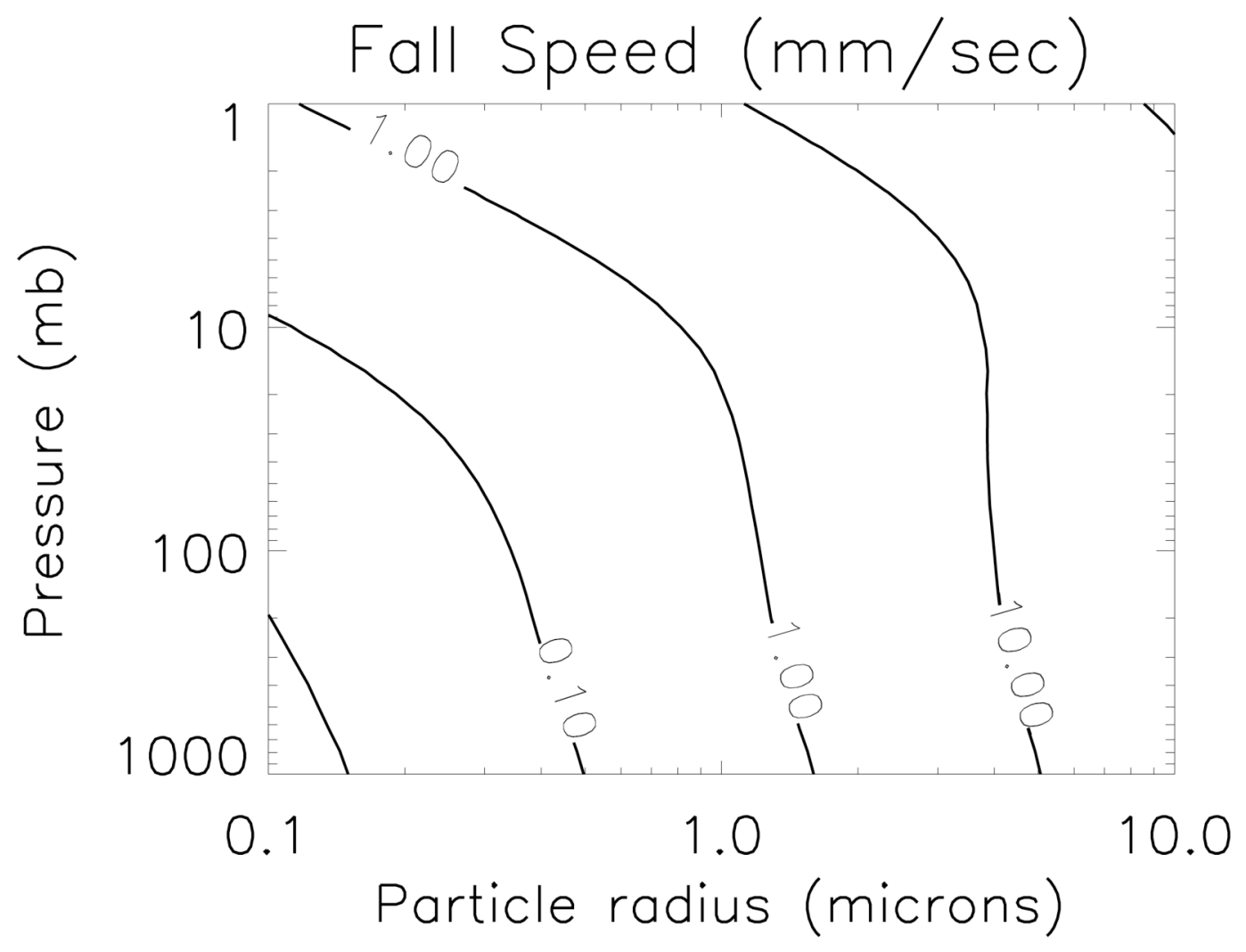

Figure 7. Fall speeds in $\mathrm{mm} / \mathrm{sec}$ for a range of particle sizes and pressures for the Saturn atmosphere at mid-latitudes. 


\subsubsection{Cloud Properties}

In contrast to the tenuous hazes that grow optically thick over a scale of kilometers, the discrete features responsible for the contrasts we measured are presumably convective condensate clouds. It is the contrast of top edges of these clouds against the thinner or relatively cloudless backgrounds that we used to determine the heights of these features. Presumably the total thickness of the features are on the order of a scale height or less, so the heights retrieved are roughly representative of the cloud's base pressure as well in most cases (albeit remarkable convective events have produced features extending far greater heights, e.g. Acarreta et al., 1999, Fischer et al., 2007). The heights inferred from the 40 different features we examined ranged from around 900 mbar at the highest to a few cases reaching the limit of detection at $\sim 2.5$ bar. The mean retrieved height is $\sim 1.75$ bar, which is

remarkably consistent with the thermochemical equilibrium model predicted height of the ammonia cloud level. Atreya et al. (1999) predicts an NH3 cloud at 1.47 bar for a solar abundance and 1.81 bar assuming a $5 x$ solar abundance of condensable volatiles; if a majority of the features detected are ammonia clouds, this suggest the abundance is closer to $5 \mathrm{x}$ solar abundance. Since these features are the tracers for zonal velocity measurements, velocity profiles show the winds in the 1 to 2 bar region.

Ammonium Hydrosulfide is predicted to form the next layer below, condensing at pressure ranging between 4.5 and 5.7 bar for the given abundances. Some of the deepest features we detect, like those in Figure 3 (feature "b"), are detectable mainly in the CB2 band and thus located at $\sim 2.5$ bar and deeper (potentially up to 6 bar if the atmosphere is clear below). The absence of similar contrast in the 
CB3 image is possibly due to the increased attenuation in the haze resulting from a relatively lower $\varpi_{0}$ at $938 \mathrm{~nm}$. Since this is well below the expected range for ammonia clouds, these deepest features may suggest significantly greater ammonia abundances than expected or the more likely detection of the putative ammonium hydrosulfide clouds predicted by models. These deeper features are likely the same clouds inferred from the VIMS spectra by Fletcher et al. (2011), located at pressures $>2$, with optical properties best fit by $\mathrm{NH}_{4} \mathrm{SH}$ (though the composition could not be identified unambiguously). These deepest clouds can only be seen in the ISS data in regions where the haze is relatively thin.

Considered with the VIMS results, our interpretation of the ISS data suggest that a majority of the opacity observed in ISS images is due to the hazes above $\sim 600$ mbar along with scattered concentrated clouds producing contrast primarily between $\sim 1$ to 2 bar. In some cases, but not always, these clouds may be optically thick enough to have significant opacity at thermal wavelengths and thus correlate with some of the observed contrast in VIMS maps. Occasional deeper clouds are detected below $\sim 2.5$ bar in regions where the haze is thin and higher clouds are absent; these are likely the concentrated clouds responsible for a majority of opacity and feature contrast at $5 \mu \mathrm{m}$, as inferred from VIMS analysis (Fletcher et al., 2011). Overlying haze and clouds obscures a majority of this deeper contrast in the ISS images. 


\subsection{Meridional Results}

Features were sampled in all major zonal regions covering a majority of planetographic latitudes between $1^{\circ} \mathrm{S}$ and $64^{\circ} \mathrm{S}$. Retrieved structures were compared to results at similar latitudes and evaluated in the context of full center-to-limb curves. We found that in general the hazes showed only little variation with longitude, and the modeled center-to-limb curves were in general in good agreement with structures inferred from the higher spatial resolution retrievals. Representative structures for each region are presented in a meridional cross section shown in Figure 8. In most cases, multiple retrieved vertical structures (with little variability) for the same latitude were averaged together to provide representative zonal averages for hazes. Zonal regions roughly corresponding to the latitude of the retrieved structures are marked on mapped MT3 $(889 \mathrm{~nm})$ and corresponding CB2 $(750 \mathrm{~nm})$ images, tilted for perspective.

From the figure, there appears to be a strong correlation between the observed reflectivity at MT3 and the height of the tropospheric haze. The structures show that regions that appear bright in the strong methane bands do so because the denser tropospheric haze extends to greater heights at these locations. This is most pronounced over the equatorial regions, where the tropospheric haze reaches up to 40 mbar. In contrast, the same haze in dark belts only reaches up to the 145 mbar level.

The tropospheric optical depth also correlates well with the observed reflectivity, but does so better in the continuum bands images. The regional differences in brightness in continuum images are thus best accounted for by increases in the optical depth of tropospheric haze. For example, though the uppermost extent of the tropospheric haze and its total optical depth are well correlated at most latitudes, 


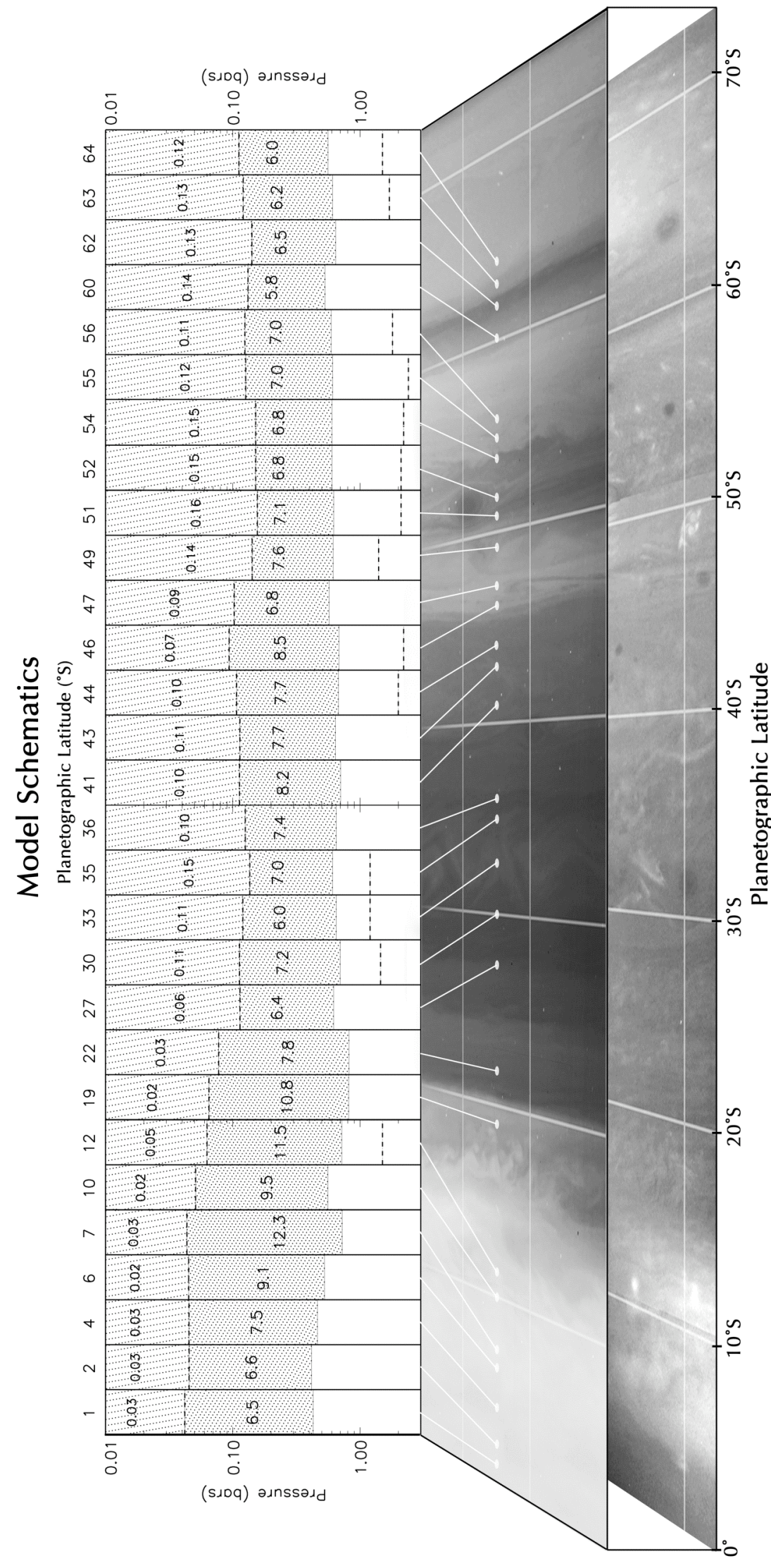

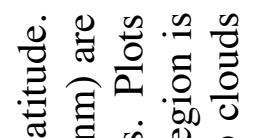

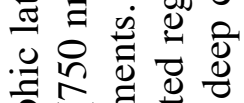

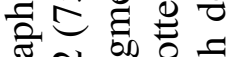

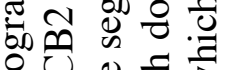

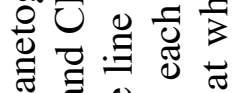

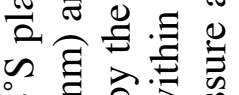

पे

0 㐫

的急

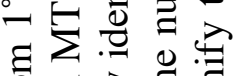

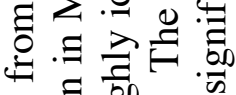

记

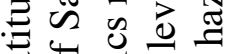

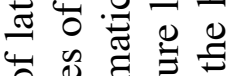

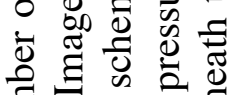

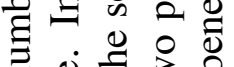

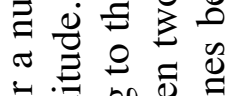

ᄒ․ㅠ

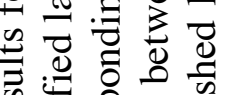

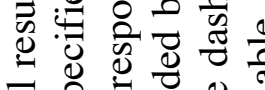

तु क

¿

छ

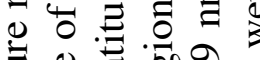

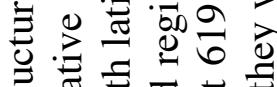

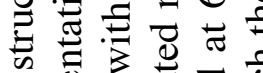

गี

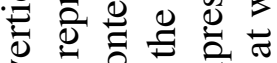
$>$ क 8 क

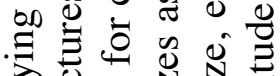

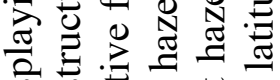

\%

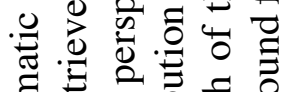

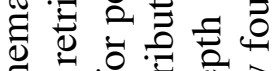

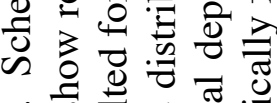

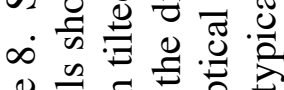

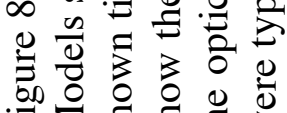

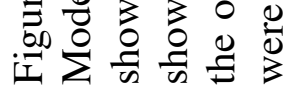


the tropospheric haze becomes optically thinner northward of $\sim 3^{\circ} \mathrm{S}$ despite reaching above 50 mbar; this is evident when comparing the methane and continua images, where the continua appear less reflective even though the methane bands maintain their high equatorial reflectivity.

Figure 9 shows a number of retrieved parameters as a function of latitude. Figures $9 \mathrm{a}$ and $9 \mathrm{~b}$ show the retrieved optical depths and vertical extent for tropospheric hazes, as discussed above. Figure 9c shows the retrieved aerosol radius, and Figure 9d and Figure 9e show the derived optical depth per bar of pressure and aerosol mixing ratios for these hazes. The maximum equatorial values are consistent with equatorial zone being a region of exceptional upwelling.

Average tropospheric particle sizes were found to be greatest towards midlatitudes, with radii around $2 \mu \mathrm{m}$ at $35^{\circ} \mathrm{S}$ gradually decreasing to the north and south. See Figure 9c. Karkoschka and Tomasko (2005) also noted such a trend. The equatorial regions had the smallest tropospheric particles with a mean slightly greater than $1 \mu \mathrm{m}$. We note that the equatorial data lacked UV3 and BL1 filters, so there was less constraint from the ratio of extinction efficiencies and scattering asymmetry; furthermore, as previously discussed, these particles sizes are at the upper limit of the range over which we can discriminate using these wavelengths. It is possible that the largest particles could in reality be even larger without significant impact on the results, but we did allow for larger radii in our model parameter space and these retrieved sizes consistently offered the best fits. 


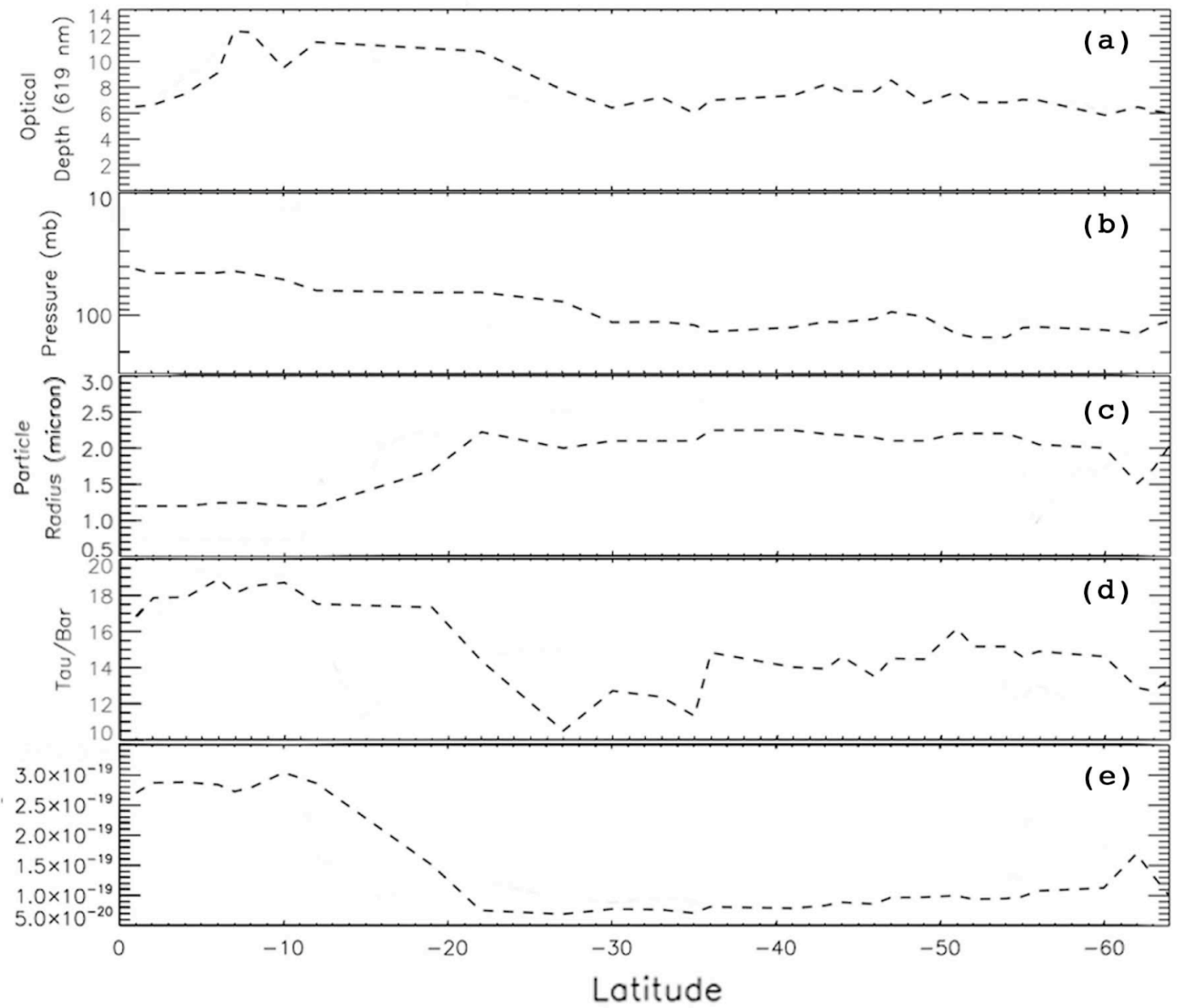

Figure 9. Retrieved model parameters as a function of planetographic latitude. (a) tropospheric haze optical depth, expressed at $619 \mathrm{~nm}$; (b) vertical extent of the tropospheric haze; (c) tropospheric haze aerosol radius; (d) derived optical depth per bar of pressure; (e) aerosol mixing ratios within the tropospheric haze. 
We did not detect any significant trends in the stratospheric particle size or particle colors for either layer between $1^{\circ} \mathrm{S}$ and $64^{\circ} \mathrm{S}$. The $\varpi_{0}$ at $451 \mathrm{~nm}$ was particularly uniform across the range, remaining close to its mean values for each layer.

We were not able to detect deep condensate clouds at all latitudes. The deepest clouds were located between about $45^{\circ} \mathrm{S}$ and $55^{\circ} \mathrm{S}$.

Latitudinal variation of the haze can be assessed in context of the temperature field. Figure 10 shows the haze distribution overlaid upon the CIRS temperature field (Fletcher et al., 2010). Also marked are the pressures at which the environmental lapse rate reaches dry adiabatic (around 500 mbar) and changes sign (around 100 mbar). Latitudes where the upper boundary of the tropospheric haze is greatest appear to roughly correspond with the regions of coldest temperatures above haze. This is consistent with the idea that these higher extending hazes are forming in regions of ascending flow accompanied by adiabatic cooling, whereas the depressed hazes are in regions of subsidence. Upwelling could be bringing more volatiles into these regions from depth, increasing the mixing ratio. The accompanying increased cooling to greater heights leads to greater condensation and higher optical depths extending to these heights. Alternatively, if the sedimentation of photochemical products from the stratosphere is the dominant source of upper tropospheric aerosols, the slight differences in weak vertical velocities would have to account for the differences in haze heights. 


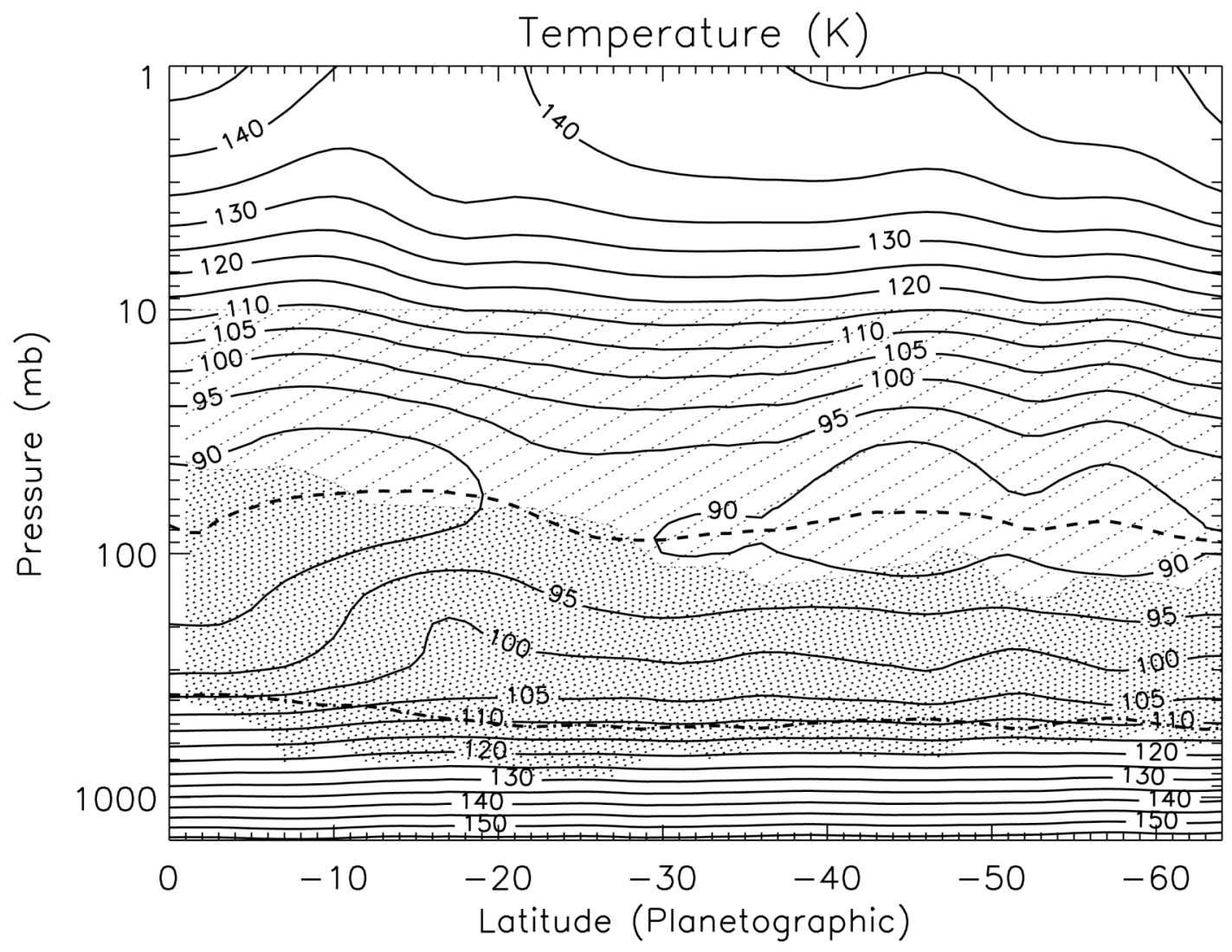

Figure10. The stratospheric and tropospheric haze distribution as a function of latitude superimposed upon a temperature field provided by Fletcher et al. (2010). The dashed line at $\sim 100$ mbar marks where the temperature lapse rate changes sign, and the dashed-dotted line at $\sim 400$ mbar marks where the lapse rate approaches dry adiabatic $(\sim 0.715 \mathrm{~K} / \mathrm{km})$. Note that the tropospheric haze exists in the stable region beneath the tropopause and above the strongly convective region. 
The latitudinal variation in the vertical extent of the haze also correlates well with the height and latitudinal trend in the implied atmospheric stability; the haze appears to reside roughly between the levels at which the environmental lapse rate is less than adiabatic (at the base) and where the temperature begins to increase with height at the tropopause.

Figure 11 illustrates the strong correlation between the upper extent of the tropospheric haze, the optical depth of the haze, and the change in temperature with pressure at a height just above the mean haze tops at $\sim 75$ mbar. Where the haze is higher and thickest, extending up above 50 mbar at roughly $10^{\circ} \mathrm{S}$, the temperature is still dropping with pressure at 50 mbar. Where the haze boundary is significantly deeper, around 140 mbar at $30^{\circ} \mathrm{S}$, the temperature has already begun increasing with height (decreasing pressure). This suggests that the extent of the haze is largely controlled by the stability and generally follows the tropopause as it changes with latitude. Since this serves as a cap on vertical transport, this also suggest that there is at least some component of the haze that is due to volatiles being transported from below.

As the particle sizes, temperature profiles, and gravity change with latitude, the fall rates of these tropospheric particles is also a function of latitude. We computed these sedimentation times as a function of latitude as shown in Figure 12, with the same assumptions as described before. The combination of largest particles under greater gravity at higher mid-latitudes results in the shortest fall-out times-less than a year to fall a scale height. In contrast, the slightly smaller particles and lesser gravity allow aerosols to fall more slowly near the equator, with one-scale height fall out 
times of more than two years. These differences may add to the greater optical depths observed in the equatorial zone with a gradually diminishing optical depths towards higher latitudes. 
Tropospheric Haze Upper Boundary, Tau \& 75-mb Lapse Rate (dT/dp)

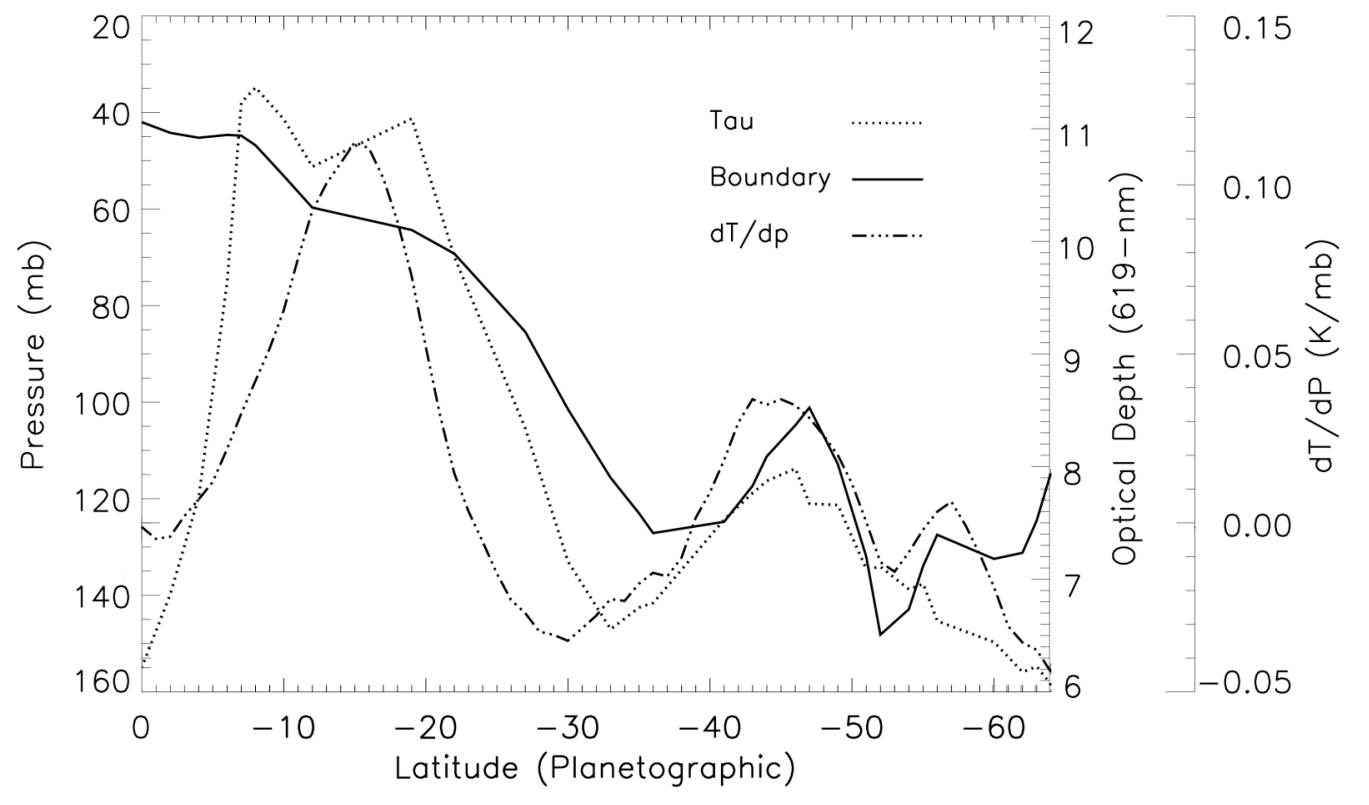

Figure11. The tropospheric haze upper extent and optical depth along with the 75mbar lapse rates (dT/dp) as a function of latitude; negative values of $\mathrm{dT} / \mathrm{dp}$ mean the temperature is increasing with height, implying strong stability. The plot shows a strong correlation between the lapse rate and haze, suggesting the extent of the haze is strongly influenced by the stability. Optical depths are expressed at 619 $\mathrm{nm}$. 


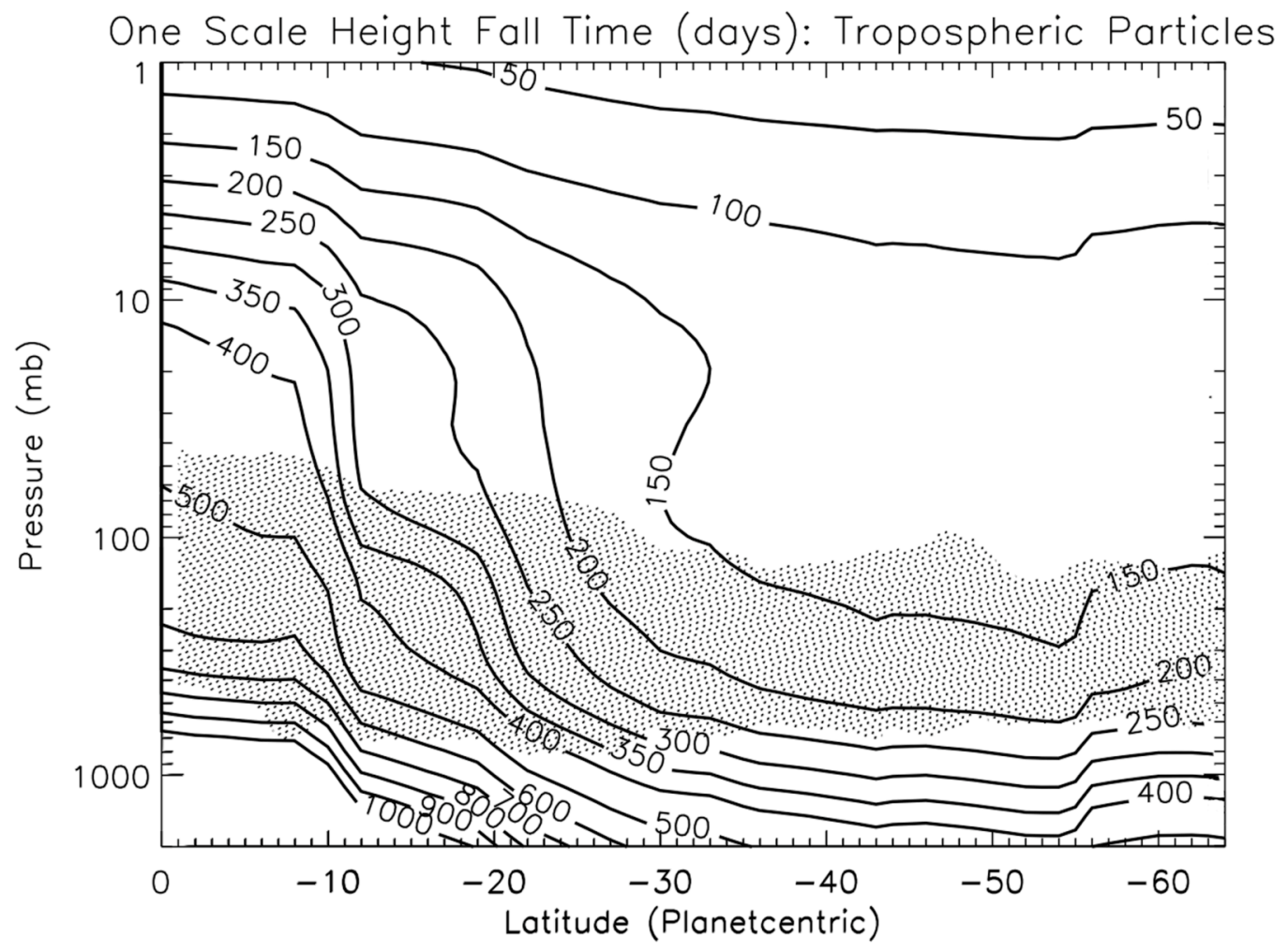

Figure 12. The fall times as a function of pressure and latitude for retrieved tropospheric haze particles sizes. Particles should remain in the atmosphere longer towards the equator given the lesser gravity and smaller particle sizes. Note that this ignores eddy diffusion or convective mixing which will dominate at lower levels. 


\subsection{Cyclone}

Finally, we examined a large cyclonic vortex located at $\sim 51^{\circ} \mathrm{S}$ planetographic latitude, featured in Figure 13. The vortex appears anomalously dark in the methane bands, and is marked by defined bright features along the northern and southern rims. This long-lived feature has been previously analyzed by Río-Gaztelurrutia et al. (2010), who investigated the vertical structure as well as the winds and dynamics.

The results of north-south and west-east sampling are presented with the locations of the retrieved structures identified in Figure 13. Once again, the relative brightness in the methane bands is attributed to the height of the troposphericstratospheric haze boundary. Both profiles show a clear trend towards a maximum in this pressure level at the center of the vortex, ranging from an average pressure of about 130 mbar to 150 mbar along the rim to a maximum pressure of about 175 mbar a the center of the vortex. This corresponds to a height difference of $\sim 5 \mathrm{~km}$ from the center to the edge of the roughly $1850 \pm 180 \mathrm{~km}$ by $860 \pm 90 \mathrm{~km}$ cyclone (RíoGaztelurrutia et al., 2010). This is consistent with this being a region of downwelling aloft, as is expected for a cyclone in Saturn's upper troposphere.

The stratospheric haze $\tau$ bar $^{-1}$ remains relatively constant at $1 \tau$ bar $^{-1}$, which is about average for the latitude. The tropospheric $\tau$ bar $^{-1}$ tends to be greater towards the center of the cyclone. Particle sizes were similar to those in neighboring regions at $0.2 \mu \mathrm{m}$ and $1.8 \mu \mathrm{m}$ for the stratospheric and tropospheric hazes, respectively, and the single scattering albedos were similar to those in neighboring regions as well.

The bright features in the center and along the rim of the vortex were attributed to deep clouds at pressures between 900 mbar and 1 bar, with the higher clouds at the 
northern and eastern edges. While the height of the tropospheric haze is in good agreement with the results of Río-Gaztelurrutia et al. (2010), our retrieved cloud heights are considerably deeper than the roughly 350 mbar cloud tops they inferred. Nevertheless, these convective cloud top heights are well above the nominal lifting condensation level of $\mathrm{NH} 3$, and this likely represents a vertically extended cloud, reaching up nearly a full scale height above most other regions. This likely indicates relatively strong convection occurring at the borders of this storm or up strong uplift due to flows impeded by the feature. There is a subtle contrast feature near the center of the oval that appears to be considerably deeper. The western part of this feature is brighter and shows greater contrast in the continuum than the eastern half. This suggests that the eastern half is a thinner cloud. However, it also shows considerably less relative contrast in the MT1 filter, consistent with it being not only thinner, but also deeper than the brighter feature to its west. Our modeling suggests the brighter, higher contrast western half is due to a thicker cloud at about 1.5 bar, whereas the eastern half appears to be located at slightly deeper than 2 bar in pressure. This could suggest a convective cloud complex with a great vertical range at depth. 

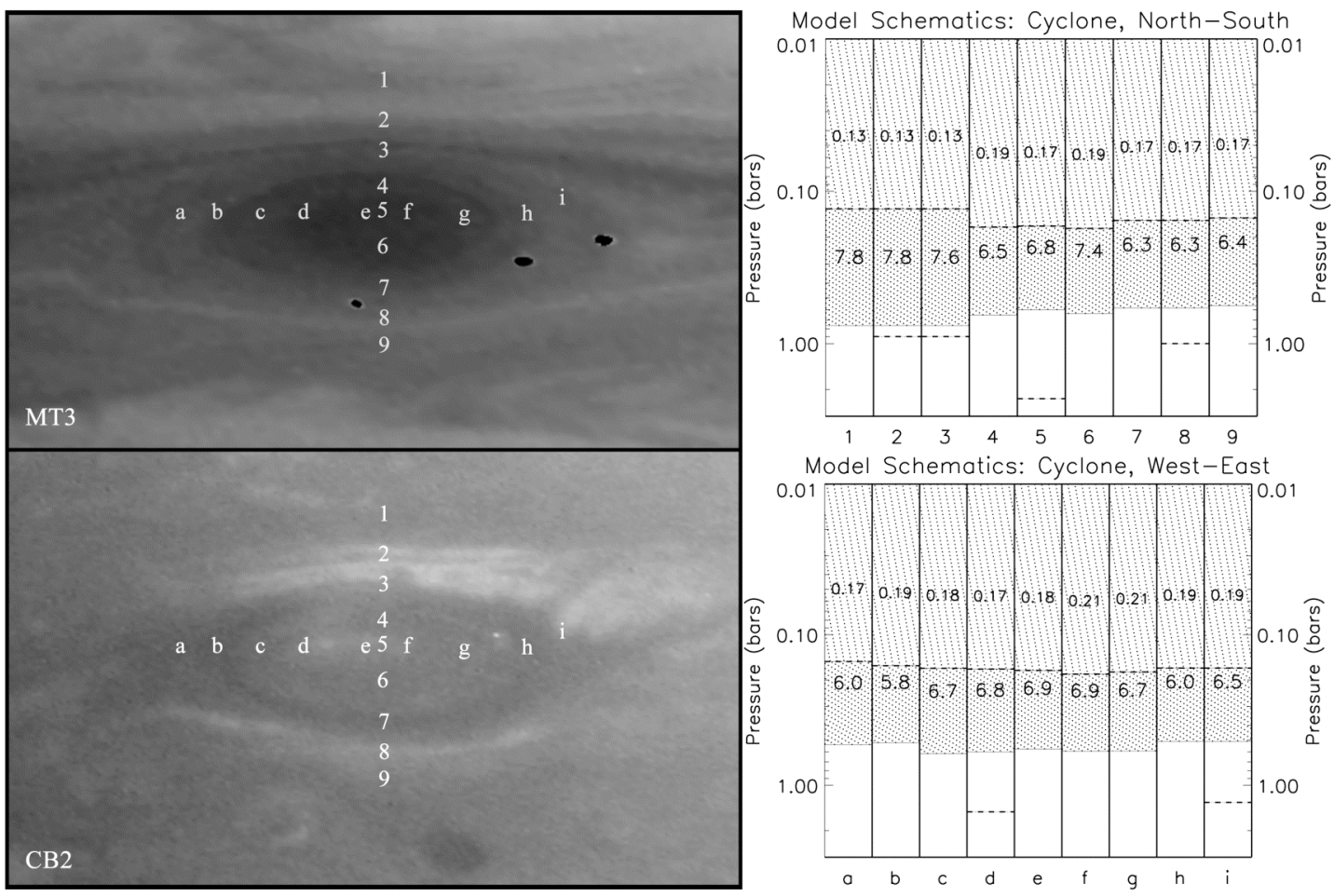

Figure13. Cyclone at $51^{\circ} \mathrm{S}$ along with schematic of retrievals. North-South and West-East transversals of the feature are annotated with corresponding numbers and letters, respectively. The vertical structure is consistent with the cyclone being a region of downwelling at the center with possibly strong uplift along the edges. 


\section{Conclusions}

Using high-resolution Cassini ISS images with wavelengths ranging from the ultraviolet to the near infrared, we retrieved atmospheric aerosol structure and properties for a range of latitudes. The observations are consistent with two distinct layers of extended hazes characterized by their vertical extent, optical depths and particle sizes all of which vary with latitude. The optically thin stratospheric haze $(\tau$ $\sim 0.02$ to $\sim 0.15 \pm 0.05$ expressed at $619 \mathrm{~nm})$ is composed of small particles $(\sim 0.3 \pm$ $0.03 \mu \mathrm{m}$ in radius), which slowly settle out over decades. The thick tropospheric haze marks an order of magnitude increase in optical depth $(\sim 6$ to $\sim 12 \pm 1 \tau)$ as larger particles $(\sim 1.8 \pm 0.4 \mu \mathrm{m})$ fall out on time scales ranging from months to years. The tropospheric haze reaches the greatest heights $(\sim 40 \pm 20$ mbar $)$ over the equator, with significantly greater depths $(\sim 140 \pm 20$ mbar $)$ at mid-latitudes, correlating well with the stability suggested by the temperature field.

Beneath these hazes, denser convective clouds create the distinct contrast features visible in continua images. A majority of these clouds are between 1 bar and 2 bar (with a mean of $1.75 \pm 0.4$ bar), serving as the tracers of winds at these heights. At high-resolution, these clouds appear broken and patchy as opposed to uniform in most locations. Occasional clouds deeper than $\sim 2.5$ bar were detected and may be visual evidence of a deeper cloud layer as inferred by VIMS analysis (Fletcher et al., 2011). These clouds are deeper than those reported in previous cloud studies using visible and near-IR wavelengths. 
Overall, our results are roughly consistent with previous findings, as well as the VIMS analysis, and offer new constraints upon of the height of the discrete cloud features.

It was our goal to build the simplest possible models capable of matching the observations, making adjustments and adding complications only as required to match our data, while making as few basic assumptions as possible. Knowledge of Saturn's hazes has been gradually inferred from a number of remote sensing studies applying observations over a range of times, geometries and wavelengths. Considering that the clouds and hazes that define Saturn's atmosphere are in reality significantly more variable and nebulous by definition than simplistic models, the amount of agreement among these studies is encouraging. 


\section{REFERENCES}

Acarreta, J.R., Sánchez-Lavega, A., 1999. Vertical cloud structure in Saturn's 1990 equatorial storm. Icarus 137, 24-33.

Atreya, S.K., Wong, M.H., Owen, T.C., Mahaffy, P.R., Niemann, H.B., de Pater, I., Drossart, P., Encrenaz, Th., 1999. Planetary and Space Science 47, 1243-1262.

Baines, K.H., Delitsky, M.L., Momary, T.W., Brown, R.H., Buratti, B.J., Clark, R.N., Nicholson, P.D., 2009. Storm clouds on Saturn: Lightning-induced chemistry and associated materials consistent with Cassini/VIMS spectra. Planetary and Space Sciences 57, 1650-1658.

Banfield, D., Gierasch, P.J., Bell, M., Ustinov, E., Ingersoll, A.P., Vasavada, A.R., West, R.A., Belton, M.J.S., 1998. Jupiter's cloud structure fromGalileo imaging data. Icarus 135, 230-250.

Borysow, A., 2002. Collision induced absorption coefficients of $\mathrm{H} 2$ pairs at temperatures from $60 \mathrm{~K}$ to $1000 \mathrm{~K}$. Astron. \& Astrophys. 390, 779-782.

Choi, D.S., Showman, A.P., Brown, R.H., 2009. Cloud features and zonal wind measurements of Saturn's atmosphere as observed by Cassini/VIMS. J. Geophys. Res., 114, E04007, doi:10.1029/2008JE003254.

Chamberlain, J.W., 1970. Behavior of Absorption Lines in a Hazy Planetary Atmosphere. Astrophysical Journal 159, 137-158.

Conrath, B.J., Gierasch, P.J., Leroy, S.S., 1990. Temperature and circulation in the stratosphere of the outer planets. Icarus 83, 255-281.

Fischer, G., Kurth, W.S., Dyudina, U.A., Kaiser, M.L., Zarka, P., Lecacheux, A., Ingersoll, A.P., Gurnett, D.A., 2007. Analysis of a giant lightning storm on Saturn. Icarus 190, 528-544.

Fletcher, L.N., Irwin, P.G.J., Teanby, N.A., Orton, G.S., Parrish, P.D., de Kok, R., Howett, C., Calcutt, S.B., Bowles, N., Taylor, F.W., 2007. Characterising Saturn's vertical temperature structure from Cassini/CIRS. Icarus 189, 457478.

Fletcher, L.N., Orton, G.S., Teanby, N.A., Irwin, P.G.J., Bjoraker, G.L., 2009. Methane and its isotopologues on Saturn from Cassini/CIRS observations. Icarus 199, 351-367. 
Fletcher, L.N., Achterberg, R.K., Greathouse, T.K., Orton, G.S., Conrath, B.J., SimonMiller, A.A., Teanby, N., Guerlet, S., Irwin, P.G.J., Flasar, F.M., 2010. Seasonal Change on Saturn from Cassini/CIRS 2004-2009, Icarus 208, 337352.

Fletcher, L.N., Baines, K.H., Momary, T.W., Showman, A.P., Irwin, P.G.J., Orton, G.S.,

Roos-Serote, M., Merlet, C., 2011. Saturn's tropospheric composition and clouds from Cassini/VIMS 4.6-5.1 $\mu \mathrm{m}$ nightside spectroscopy. Icarus 214, 510-533.

Hansen, J. H., and L. D. Travis 1974. Light scattering in planetary atmospheres. Space Sci. Rev. 16, 527-610.

Irwin, P.G., Weir, A.L., Taylor, F.W., Calcutt, S.B., 2001. The origin of belt/zone contrasts in the atmosphere of Jupiter and the their correlation with 5- $\mu \mathrm{m}$ opacity. Icarus $149,397-415$

Jacobson, R.A., 2004. The orbits of the major Saturnian satellites and the gravity field of Saturn from spacecraft and Earth-based observations. Astron. J. 128, 492501 .

Karkoschka, E., Tomasko, M.G., 2005. Saturn's vertical and latitudinal cloud structure 1991-2004 from HST imaging in 30 filters. Icarus 179, 195-221

Karkoschka E., and M. G. Tomasko 2010. Methane absorption coefficients for the Jovian planets from laboratory, Huygens, and HST data. Icarus. 205, 674-694.

Lindal, G.F., Sweetnam, D.N., and Eshleman, V.R., 1985. The atmosphere of Saturn: An analysis of the Voyager radio occultation measurements. Astron. J. 90, 1136-1146.

Lewis, J.S. 1969. The clouds of Jupiter and the $\mathrm{NH}_{3}-\mathrm{H}_{2} \mathrm{O}$ and $\mathrm{NH}_{3}-\mathrm{H}_{2} \mathrm{~S}$ systems. Icarus 10, 365-378.

Martonchik, J. V., G. S. Orton, and J. F.Appleby 1984.Optical properties of NH3 ice from the far infrared to the near ultraviolet. Appl. Opt. 23, 541-547.

Moses, J.I., 2000. Photochemistry of Saturn's atmosphere. Icarus 143, 244-298.

Muñoz, O., Morena, F., Molina, A., Grodent, D., Gérard, Dols, V., 2004. Study of the vertical structure of Saturn's atmosphere using HST/WFPC2 images. Icarus 169, 413-428. 
Pérez-Hoyos, S., Sánchez-Lavega, A., French, R.G., Rojas, J.F., 2005. Saturn's cloud structure and temporal evolution fomr ten years of Hubble Space Telescope Images (1994-2003). Icarus 176, 155-174.

Porco, C., West, R., McEwen, A., Del Genio, A., Ingersoll, A., Thomas, P., Squyres, S., Dones, L., Murray, C., Johnson, T., Burns, J., Brahic, A., Neukum, G., Veverka, J., Barbara, J., Denk, T., Evans, M., Ferrier, J., Geissler, P., Helfenstein, P., Roatsch, T., Throop, H., Tiscareno, M., Vasavada, A., 2003. Cassini imaging of Jupiter's atmosphere, satellites, and rings. Science 299, 1541-1547.

del Río-Gaztelurrutia, T., Legarreta, J., Hueso, R., Pérez-Hoyos, S., Sánchez-Lavega, A., 2010. A long-lived cyclone in Saturn's atmosphere: Observations and models. Icarus 209, 665-681.

Sánchez-Lavega, A., Hueso, R.,Pérez-Hoyos, S., 2007. The three dimensional structure of Saturn's equatorial jet at cloud level. Icarus 187, 510-519.

Simon-Miller, A. A., D. Banfield, and P. J. Gierasch 2001. Color and the vertical structure in Jupiter's belts, zones, and weather systems. Icarus 154, 459-474.

Stam, D.M., Banfield, D., Gierasch, P.J., Nicholson, P.D., Matthews, K., 2001. NearIR spectrophotometry of saturnian aerosols - meridional and vertical distribution. Icarus 152. 407-422

Temma, T., Chanover, N.J., Simon-Miller, A.A., Glenar, D.A., Hillman, J.J., Kuehn, D.M., 2005. Vertical structure modeling of Saturn's equatorial region using high spectral resolution imaging. Icarus 175, 464-489.

Tomasko, M.G., Bézard, B., Doose, L., Engel, S., Karkoschka, E., 2008 b. Measurements of methane absorption by the descent imager/spectral radiometer (DISR) during its descent through Titan's atmosphere. Planet. Space Sci. 56, 624-647.

Vasavada, A.R., Hörst, Kennedy, M.R., Ingersoll, A.P., Porco, C.C., Del Genio, A.D., West, R.A., 2006. Cassini imaging of Saturn: Southern hemisphere winds and vortices. J. Geophys. Res., 111, E05004, doi:10.1029/2005JE002563.

Weidenschilling, S.J., Lewis, J.S., 1973. Atmospheric and cloud structures on the jovian planets. Icarus 20, 465-476.

West, R.A., Friedson, A.J., and Appleby, J.F., 1992. Jovian large-scale stratospheric circulation. Icarus 100, 245-259. 
West, R.A., Baines, K.H., Friedson, A.J., Banfield, D., Ragent, B., Taylor,F.W., 2004. Jovian clouds and haze. In: Bagenal, F., Dowling, T., McKinnon, W. (Eds.), Jupiter: The Planet, Satellites, and Magnetosphere. Cambridge Univ. Press, Cambridge, 79-104.

West, R.A., Baines, K.H., Karkoschka, E., Sánchez-Lavega, A., 2009. Clouds and aerosols in Saturn's atmosphere, In: Dougherty, M.K., Esposito, L.W., Krimigis, S.M. (Eds.), Saturn from Cassini-Huygens. Springer Netherlands, pp. 161-179.

West, R., B. Knowles, E. Birath, S. Charnoz, D. Di Nino, M. Hedman, P. Helfenstein, A. McEwen, J. Perry, C. Porco, J. Salmon, H. Throop, D. Wilson 2010. Inflight calibration of the Cassini imaging science sub-system cameras.

Planetary and Space Sci. 58, 1475-1488. 